\title{
Structure and Activity of the Camellia Sapogenin Derivatives on Biofilm Inhibition of Staphylococcus aureus and Escherichia coli
}

Chunfang Zhu ${ }^{1}$, Meng Zhang ${ }^{1}$, Qiaoling Tang ${ }^{1}$, Qian Yang ${ }^{1}$, Yong Ye ${ }^{1,2^{*}}$

${ }^{1}$ Department of Pharmaceutical Engineering, School of Chemistry and Chemical Engineering, South

China University of Technology, Guangzhou 510640, People's Republic of China

${ }^{2}$ Gannan Medical University Collaborative Innovation Center for Gannan Oil-tea Camellia Industrial

Development, Guanzhou 341000, P R China

*yeyong@scut.edu.cn

\section{Contents}

Synthesis of key intermediates.

Synthesis of Camellia sapogenin derivative S-(1-19)

${ }^{1} \mathrm{H}$ NMR and ${ }^{13} \mathrm{C}$ NMR Spectrum for sapogenin $\left(\mathrm{C}_{2} \mathrm{D}_{6} \mathrm{SO}\right.$ as solvent $)$ 
${ }^{1} \mathrm{H}$ NMR and ${ }^{13} \mathrm{C}$ NMR Spectrum for Camellia sapogenin derivative S-(1-19) $\left(\mathrm{C}_{2} \mathrm{D}_{6} \mathrm{SO}\right.$ as solvent $)$ 
Synthesis of key intermediates. The desired raw material, Camellia sapogenin, was prepared by the purification of defatted Camellia oleifera seeds and acid-base hydrolysis. Sapogenin (10.1 g, $20 \mathrm{mmol})$ and triphenylchloromethane $(5.57 \mathrm{~g}, 20 \mathrm{mmol})$ were dissolved in pyridine $(40 \mathrm{~mL})$ and reacted at room temperature for $24 \mathrm{~h}$. Then the residue was washed with water, ethyl acetate and saturated sodium chloride solution, dried with sodium sulfate, and finally evaporated to remove the organic solvent. Intermediate 1a was obtained as white solid; yield, $91.9 \%$. Then intermediate $1 \mathbf{a}(7.47 \mathrm{~g}, 10 \mathrm{mmol})$ was mixed with acetic anhydride $(3.78 \mathrm{~mL}, 40 \mathrm{mmol})$ in DMF $(40 \mathrm{~mL})$, catalyzed by triethylamine $(1.66$ $\mathrm{mL}, 12 \mathrm{mmol}$ ) at room temperature for $24 \mathrm{~h}$. The residue was washed, dried and evaporated in the same way to obtain Intermediate $\mathbf{2 a}$ as white solid; yield, $86.0 \%$. At last, methanol hydrochloride solution (5 $\mathrm{mL}, 10 \%, \mathrm{v} / \mathrm{v})$ was added to the dichloromethane / methanol solution $(1: 1, \mathrm{v} / \mathrm{v})$ of intermediate $2 \mathbf{a}$ $(4.58 \mathrm{~g}, 5 \mathrm{mmol})$, and the reaction was carried out at room temperature for $24 \mathrm{~h}$. After washing and drying, it is purified by column chromatography to obtain intermediate 3 a as white solid; yield, $82.3 \%$.

\section{Synthesis of Camellia sapogenin derivative S-(1-19).}

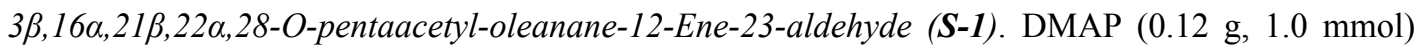
and intermediate 3a $(0.67 \mathrm{~g}, 1.0 \mathrm{mmol})$ were dissolved in DMSO $(10 \mathrm{~mL})$ and reacted with acetic anhydride $(115 \mu \mathrm{L}, 1.2 \mathrm{mmol})$ at $40^{\circ} \mathrm{C}$ for $24 \mathrm{~h}$. After the reaction, the resulting mixture was diluted with distilled water and extracted with ethyl acetate. The residue was washed with hydrochloric acid, saturated sodium carbonate and saturated sodium chloride, dried with anhydrous sodium sulfate and evaporated to remove the organic solvent under reduced pressure, the final Camellia sapogenin derivative S-1 was obtained as white solid (0.65 g, 90.92\%). IR (KBr) 3360, 2933, 2861, 1739, 1657 $\mathrm{cm}^{-1} ;{ }^{1} \mathrm{H}$ NMR (400 MHz, $\left.\mathrm{C}_{2} \mathrm{D}_{6} \mathrm{SO}\right) \delta: 9.29$ (s, 1H, CHO), 5.57 (d, J=8.0 Hz, 1H, 21-H), 5.19 (t, 1H, 12-H), $5.13(\mathrm{~d}, J=12.1 \mathrm{~Hz}, 1 \mathrm{H}, 22-\mathrm{H}), 4.35(\mathrm{t}, J=5.1 \mathrm{~Hz}, 1 \mathrm{H}, 3-\mathrm{H}), 4.19(\mathrm{~m}, 1 \mathrm{H}, 16-\mathrm{H}), 3.96(\mathrm{~d}, J=$ $11.3 \mathrm{~Hz}, 1 \mathrm{H}, 28-\mathrm{H}), 3.87(\mathrm{~d}, J=11.7 \mathrm{~Hz}, 1 \mathrm{H}, 28-\mathrm{H}), 1.99\left(\mathrm{~s}, 15 \mathrm{H}, \mathrm{CH}_{3}\right), 1.92(\mathrm{t}, J=6.6 \mathrm{~Hz}, 1 \mathrm{H}, 18-\mathrm{H})$, 1.85-1.30 (m, 16H, CH $), 1.26\left(\mathrm{~s}, 3 \mathrm{H}, \mathrm{CH}_{3}, 24-\mathrm{H}\right), 1.04-0.90$ (s, 3H, 27-H), 0.90-0.73 (s, 12H, $\mathrm{CH}_{3}$, 25,26,29,30-H). ${ }^{13} \mathrm{C}$ NMR (151 MHz, $\left.\mathrm{C}_{2} \mathrm{D}_{6} \mathrm{SO}\right) \delta: 205.35,170.33,170.30,170.20,170.03,157.07$, $142.28,123.28,87.17,77.45,76.82,73.18,73.02,54.28,47.98,46.71,46.31,46.13,42.33,41.49$, $41.30,38.24 ; 37.74,35.63,33.28,31.56,27.43,25.81,25.62,23.46,22.57,21.49,21.40,21.26,21.14$, 20.97, 20.86, 17.11, 15.77, 9.66. HRMS $\left(\mathrm{ESI}^{+}\right)$, calcd for $\mathrm{C}_{40} \mathrm{H}_{59} \mathrm{O}_{11}(\mathrm{M}+\mathrm{H})^{+}, 715.4213$; found, 715.4221. 
$\mathrm{g}, 1.0 \mathrm{mmol})$ and intermediate $\mathbf{3 a}(0.67 \mathrm{~g}, 1.0 \mathrm{mmol})$ were dissolved in pyridine $(10 \mathrm{~mL})$ and reacted with chloracetyl chloride $\left(90 \mu \mathrm{L}, 1.2 \mathrm{mmol}\right.$ ) at $40^{\circ} \mathrm{C}$ for $24 \mathrm{~h}$. The post-processing was the same as that of S-1 and sapogenin derivative S-2 was obtained as white solid (0.69 g, 92.08\%). IR (KBr) 3327, 2926, 2853, 1726, 1622, $735 \mathrm{~cm}^{-1} .{ }^{1} \mathrm{H}$ NMR (400 MHz, $\left.\mathrm{C}_{2} \mathrm{D}_{6} \mathrm{SO}\right) \delta: 9.32(\mathrm{~s}, 1 \mathrm{H}, \mathrm{CHO}), 5.56(\mathrm{~d}, J=8.0 \mathrm{~Hz}$, 2H, 21,22-H), 5.32 (s, 1H, 12-H), 4.34 (t, 1H, J = 5.1 Hz, 3-H), 4.34 (s, 2H), 4.19 (s, 1H, 16-H), 3.96 (d, $J=11.3 \mathrm{~Hz}, 1 \mathrm{H}, 28-\mathrm{H}), 3.87(\mathrm{~d}, J=11.7 \mathrm{~Hz}, 1 \mathrm{H}, 28-\mathrm{H}), 1.99(\mathrm{t}, J=6.2 \mathrm{~Hz}, 1 \mathrm{H}, 18-\mathrm{H}), 1.72(\mathrm{~s}$, 12H, $\left.\mathrm{CH}_{3}\right), 1.70-1.20\left(\mathrm{~m}, 16 \mathrm{H}, \mathrm{CH}_{2}\right), 1.10-0.80\left(\mathrm{~s}, 18 \mathrm{H}, \mathrm{CH}_{3}, 24,25,26,27,29,30-\mathrm{H}\right) .{ }^{13} \mathrm{C}$ NMR $(151$ $\left.\mathrm{MHz}, \mathrm{C}_{2} \mathrm{D}_{6} \mathrm{SO}\right) \delta: 204.46,170.2,169.98,157.23,157.08,157.02,144.13,128.19,86.46,82.35,76.82$, $71.42,65.45,54.49,47.98,47.92,46.98,46.89,45.18,44.18,42.86,38.1,36.66,35.30,29.48,28.15$, $25.92,25.80,25.81,25.62,25.03,24.93,24.92,24.65,24.02,23.96,23.82,19.12,19.01,16.78$. HRMS $\left(\mathrm{ESI}^{+}\right)$, calcd for $\mathrm{C}_{40} \mathrm{H}_{58} \mathrm{O}_{11} \mathrm{Cl}(\mathrm{M}+\mathrm{H})^{+}, 749.3453$; found, 746.3482.

28-O-trifluoroacetyl-3 $\beta, 16 \alpha, 21 \beta, 22 \alpha$-O-tetraacetyl-oleanone-12-Ene-23-aldehyde $\quad(\boldsymbol{S}$-3). DMAP $(0.12 \mathrm{~g}, 1.0 \mathrm{mmol})$ and intermediate $3 \mathrm{a}(0.67 \mathrm{~g}, 1.0 \mathrm{mmol})$ were dissolved in DMSO (10 $\mathrm{mL})$ and reacted with trifluoroacetic anhydride $(170 \mu \mathrm{L}, 1.2 \mathrm{mmol})$ at $40^{\circ} \mathrm{C}$ for $24 \mathrm{~h}$. The post-processing was the same as that of S-1 and sapogenin derivative S-3 was obtained as white solid $(0.69 \mathrm{~g}, 89.74 \%)$. IR (KBr) 3329, 2926, 2864, 1778, 1729, 1624, $1084 \mathrm{~cm}^{-1} .{ }^{1} \mathrm{H}$ NMR (400 MHz, $\left.\mathrm{C}_{2} \mathrm{D}_{6} \mathrm{SO}\right) \delta: 9.26(\mathrm{~s}, 1 \mathrm{H}$, CHO), $5.57(\mathrm{~d}, 1 \mathrm{H}, 21-\mathrm{H}), 5.32(\mathrm{~d}, J=4.6 \mathrm{~Hz}, 1 \mathrm{H}, 22-\mathrm{H}), 5.19(\mathrm{t}, 1 \mathrm{H}, 12-\mathrm{H}), 4.22(\mathrm{t}, J=6.5 \mathrm{~Hz}, 1 \mathrm{H}$, 3-H), $4.16(\mathrm{~m}, 1 \mathrm{H}, 16-\mathrm{H}), 4.03-3.92(\mathrm{~d}, 2 \mathrm{H}, 28-\mathrm{H}), 1.99$ (t, J=10.2 Hz, 1H, 18-H), $1.86\left(\mathrm{~s}, 12 \mathrm{H}, \mathrm{CH}_{3}\right)$, 1.80-1.20 (m, 16H, CH 25,26,29,30-H). ${ }^{13} \mathrm{C}$ NMR (151 MHz, $\left.\mathrm{C}_{2} \mathrm{D}_{6} \mathrm{SO}\right) \delta: 207.45,171.05,164.49,157.08,157.02,150.07$, $140.06,129.11,117.34,88.16,77.24,74.26,69.07,63.56,55.28,47.98,47.27,47.01,46.86,42.36$, $41.32,39.30,38.17,34.13,33.81,31.97,31.76,30.48,30.19,29.49,29.17,29.03,25.80,25.60,25.46$, 25.32, 24.93, 22.56, 19.12, 14.39. HRMS $\left(\mathrm{ESI}^{+}\right)$, calcd for $\mathrm{C}_{40} \mathrm{H}_{56} \mathrm{O}_{11} \mathrm{~F}_{3}(\mathrm{M}+\mathrm{H})^{+}, 769.3562$; found, 769.3530.

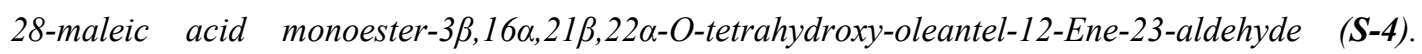
DMAP $(0.12 \mathrm{~g}, 1.0 \mathrm{mmol})$ and intermediate 3a $(0.67 \mathrm{~g}, 1.0 \mathrm{mmol})$ were dissolved in DMSO $(10 \mathrm{~mL})$ and reacted with maleic anhydride $(0.12 \mathrm{~g}, 1.2 \mathrm{mmol})$ at $40^{\circ} \mathrm{C}$ for $24 \mathrm{~h}$. After the reaction, the resulting mixture was purified by the same method as $\mathbf{S - 1}$ and then dissolved in dichloromethane / methanol (1: 1, v: v) to react with acetylchloride $(11 \mu \mathrm{L}, 0.15 \mathrm{mmol})$ at room temperature for $6 \mathrm{~h}$. The solvent was removed and the product was extracted with ethyl acetate, and the ethyl acetate was removed under 
reduced pressure. Sapogenin derivative S-4 was obtained as white solid (0.5 g, 82.95\%). IR (KBr) 3337, 2931, 2864, 1718, 1655, $720 \mathrm{~cm}^{-1} .{ }^{1} \mathrm{H}$ NMR (400 MHz, $\left.\mathrm{C}_{2} \mathrm{D}_{6} \mathrm{SO}\right) \delta: 12.48(\mathrm{~s}, 1 \mathrm{H}, \mathrm{COOH}), 9.38(\mathrm{~s}, 1 \mathrm{H}$, CHO), 6.94 (s, 1H, OH, 3-H), $6.44(\mathrm{~d}, J=2.1 \mathrm{~Hz}, 1 \mathrm{H}, \mathrm{CH}), 6.19(\mathrm{~d}, J=2.1 \mathrm{~Hz}, 1 \mathrm{H}, \mathrm{CH}), 5.32(\mathrm{~s}, 2 \mathrm{H}$, OH, 21,22-H), $5.18(\mathrm{t}, 1 \mathrm{H}, 12-\mathrm{H}), 5.04(\mathrm{~s}, 1 \mathrm{H}, 16-\mathrm{OH}), 4.17(\mathrm{~d}, J=3.3 \mathrm{~Hz}, 1 \mathrm{H}, 28-\mathrm{H}), 3.97(\mathrm{~m}, 1 \mathrm{H}$, 3-H), $3.89(\mathrm{~d}, \mathrm{~J}=6.5 \mathrm{~Hz}, 1 \mathrm{H}, 28-\mathrm{H}), 3.35-3.30(\mathrm{~m}, 3 \mathrm{H}, 16,21,22-\mathrm{H}), 1.99(\mathrm{t}, \mathrm{J}=12.0 \mathrm{~Hz}, 1 \mathrm{H}, 18-\mathrm{H})$, 1.90-1.30 (m, 16H, $\left.\mathrm{CH}_{2}\right), 1.23$ (s, 3H, CH $\left.\mathrm{CH}_{3}, 24-\mathrm{H}\right), 1.06$ (s, 3H, 27-H), 0.95-0.82(s, 12H, $\mathrm{CH}_{3}, 25,26$ 29,30-H). ${ }^{13} \mathrm{C}$ NMR (151 MHz, $\left.\mathrm{C}_{2} \mathrm{D}_{6} \mathrm{SO}\right) \delta: 207.50,164.35,161.18,144.01,136.13,129.94,122.14$, 77.30, 71.63, 71.56, 70.92, 67.60, 55.59, 47.41, 46.82, 46.65, 46.48, 44.06, 41.73, 41.71, 38.88, 36.72, $35.70,33.99,31.59,27.47,25.45,25.42,23.44,22.57,21.33,16.83,15.95,9.35$. HRMS $\left(\right.$ ESI $^{+}$), calcd for $\mathrm{C}_{34} \mathrm{H}_{51} \mathrm{O}_{9}(\mathrm{M}+\mathrm{H})^{+}, 603.3568$; found, 603.3562 .

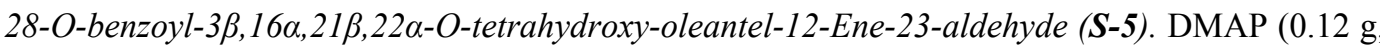
$1.0 \mathrm{mmol})$ and intermediate $3 \mathrm{a}(0.67 \mathrm{~g}, 1.0 \mathrm{mmol})$ were dissolved in pyridine $(10 \mathrm{~mL})$ and reacted with benzoyl chloride $(115 \mu \mathrm{L}, 1.2 \mathrm{mmol})$ at $40^{\circ} \mathrm{C}$ for $24 \mathrm{~h}$. The post-processing was the same as that of $\mathbf{S}-4$ and sapogenin derivative S-5 was obtained as light-yellow powder (0.51 g, 83.77\%). IR (KBr) 3535, $3065,2938,2858,1719,1595,1525,1456,1380,711.5 \mathrm{~cm}^{-1} .{ }^{1} \mathrm{H}$ NMR (400 MHz, $\left.\mathrm{C}_{2} \mathrm{D}_{6} \mathrm{SO}\right) \delta: 9.42(\mathrm{~s}$, $1 \mathrm{H}, \mathrm{CHO}), 7.99(\mathrm{~d}, 2 \mathrm{H}), 7.76\left(\mathrm{dd}, J_{1}=8.5 \mathrm{~Hz}, J_{2}=1.7 \mathrm{~Hz}, 1 \mathrm{H}\right), 7.55(\mathrm{~m}, J=7.6 \mathrm{~Hz}, 2 \mathrm{H}), 6.74(\mathrm{~s}, 1 \mathrm{H}$, OH, 3-H), $5.58(\mathrm{~s}, 1 \mathrm{H}, \mathrm{OH}, 21-\mathrm{H}), 5.55$ (s, 1H, OH, 22-H), 5.07 (t, 1H, 12-H), $4.64(\mathrm{~s}, 1 \mathrm{H}, \mathrm{OH}, 16-\mathrm{H})$, $4.31(\mathrm{~d}, J=18.3 \mathrm{~Hz}, 1 \mathrm{H}, 28-\mathrm{H}), 4.02(\mathrm{~d}, J=12.3 \mathrm{~Hz}, 1 \mathrm{H}, 28-\mathrm{H}), 3.83(\mathrm{t}, 1 \mathrm{H}, 3-\mathrm{H}), 3.70-3.51(\mathrm{~m}, 3 \mathrm{H}$, 16,21,22-H), $1.91(\mathrm{t}, J=4.5 \mathrm{~Hz}, 1 \mathrm{H}, 18-\mathrm{H}), 1.87-1.30\left(\mathrm{~m}, 16 \mathrm{H}, \mathrm{CH}_{2}\right), 1.25$ (s, 3H, $\left.\mathrm{CH}_{3}, 24-\mathrm{H}\right), 1.09-$ 0.81 (s, $\left.15 \mathrm{H}, \mathrm{CH}_{3}, 25,26,27,29,30-\mathrm{H}\right) .{ }^{13} \mathrm{C}$ NMR (151 MHz, $\left.\mathrm{C}_{2} \mathrm{D}_{6} \mathrm{SO}\right) \delta: 205.50,165.79,142.53$, $133.91,130.79,129.78,129.74,128.96,128.91,123.41,81.38,74.70,73.99,68.63,67.93,54.56,47.05$, $46.69,46.34,46.15,43.04,41.79,41.48,38.18,36.86,35.70,33.31,31.77,27.43,25.07,25.02,23.47$, 22.56, 20.53, 17.28, 15.78, 9.79. HRMS $\left(\mathrm{ESI}^{+}\right)$, calcd for $\mathrm{C}_{37} \mathrm{H}_{53} \mathrm{O}_{7}(\mathrm{M}+\mathrm{H})^{+}, 609.5747$; found, 609.5750.

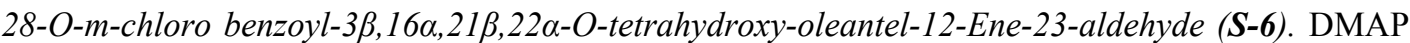
$(0.12 \mathrm{~g}, 1.0 \mathrm{mmol})$ and intermediate $3 \mathrm{a}(0.67 \mathrm{~g}, 1.0 \mathrm{mmol})$ were dissolved in pyridine $(10 \mathrm{~mL})$ and reacted with 3-chlorophenyl methyl chloride $(155 \mu \mathrm{L}, 1.2 \mathrm{mmol})$ at $40^{\circ} \mathrm{C}$ for $24 \mathrm{~h}$. The post-processing was the same as that of S-4 and sapogenin derivative S-6 was obtained as light-yellow powder $(0.51 \mathrm{~g}$, 79.28\%). IR (KBr) 3440, 3070, 1642, 1572, 1532, 1462, 1120, 1093, 890, 798, $741 \mathrm{~cm}^{-1} .{ }^{1} \mathrm{H}$ NMR (400 $\left.\mathrm{MHz}, \mathrm{C}_{2} \mathrm{D}_{6} \mathrm{SO}\right) \delta: 9.39(\mathrm{~s}, 1 \mathrm{H}, \mathrm{CHO}), 7.96(\mathrm{~s}, 1 \mathrm{H}), 7.89(\mathrm{~d}, J=7.9 \mathrm{~Hz}, 1 \mathrm{H}), 7.52(\mathrm{~d}, J=7.8 \mathrm{~Hz}, 1 \mathrm{H})$, 
$7.41(\mathrm{~m}, J=2.3 \mathrm{~Hz}, 1 \mathrm{H}), 6.20(\mathrm{~s}, 1 \mathrm{H}, \mathrm{OH}, 3-\mathrm{H}), 5.77$ (s, 1H, OH, 21-H), 5.75 (s, 1H, OH, 22-H), 5.45 (s, 1H, OH, 16-H), 5.14 (t, 1H, 12-H), 4.33 (d, $J=5.1 \mathrm{~Hz}, 1 \mathrm{H}, 28-\mathrm{H}), 3.96(\mathrm{~d}, J=7.0 \mathrm{~Hz}, 1 \mathrm{H}, 28-\mathrm{H})$, 3.60-3.40 (m, 4H, 3,16,21,22-H), 2.18 (t, $J=7.2 \mathrm{~Hz}, 1 \mathrm{H}, 18-\mathrm{H}), 1.90-1.20\left(\mathrm{~m}, 16 \mathrm{H}, \mathrm{CH}_{2}\right), 1.15-0.70$ (s, $\left.18 \mathrm{H}, \mathrm{CH}_{3}, 24,25,26,27,29,30-\mathrm{H}\right) .{ }^{13} \mathrm{C}$ NMR (151 MHz, $\left.\mathrm{C}_{2} \mathrm{D}_{6} \mathrm{SO}\right) \delta: 208.44,166.89,139.61,134.00$, $133.86,131.92,130.88,129.28,128.99,126.05,74.60,73.56,71.13,65.50,65.45,54.12,49.54,48.69$, $48.36,48.15,45.04,43.79,42.38,38.14,36.81,35.69,33.23,30.49,27.04,25.57,25.43,23.44,22.56$, 19.12, 17.42, 13.99, 9.74. HRMS (ESI+), calcd for $\mathrm{C}_{37} \mathrm{H}_{52} \mathrm{O}_{7} \mathrm{Cl}(\mathrm{M}+\mathrm{H})^{+}, 643.3362$; found, 643.3364 .

28-O-m-fluoro benzoyl-3 $\beta, 16 \alpha, 21 \beta, 22 \alpha$-O-tetrahydroxy-oleantel-12-Ene-23-aldehyde (S-7). DMAP $(0.12 \mathrm{~g}, 1.0 \mathrm{mmol})$ and intermediate $3 \mathrm{a}(0.67 \mathrm{~g}, 1.0 \mathrm{mmol})$ were dissolved in pyridine $(10 \mathrm{~mL})$ and reacted with 3 -fluorobenzophenyl chloride $(145 \mu \mathrm{L}, 1.2 \mathrm{mmol})$ at $40^{\circ} \mathrm{C}$ for $24 \mathrm{~h}$. The post-processing was the same as that of S-4 and sapogenin derivative S-7 was obtained as light-yellow powder $(0.49 \mathrm{~g}$, 78.18\%). IR (KBr) 3442, 3074, 2928, 2858, 1720, 1644, 1589, 1535, 1480, 1201; 1084, 971, 888, 801, 751, $677 \mathrm{~cm}^{-1} .{ }^{1} \mathrm{H}$ NMR (400 MHz, $\left.\mathrm{C}_{2} \mathrm{D}_{6} \mathrm{SO}\right) \delta: 9.40$ (s, 1H, CHO), 7.95 (s, 1H), 7.79 (d, J= $7.2 \mathrm{~Hz}$, 1H), $7.58(\mathrm{~s}, J=8.0 \mathrm{~Hz}, 1 \mathrm{H}), 7.29$ (d, $J=8.1 \mathrm{~Hz}, 1 \mathrm{H}), 6.29$ (s, 1H, OH, 3-H), 5.35 (s, 1H, OH, 21-H), $5.26(\mathrm{~s}, 1 \mathrm{H}, \mathrm{OH}, 22-\mathrm{H}), 5.14(\mathrm{t}, 1 \mathrm{H}, 12-\mathrm{H}), 4.61(\mathrm{~s}, 1 \mathrm{H}, \mathrm{OH}, 16-\mathrm{H}), 4.34(\mathrm{t}, J=5.0 \mathrm{~Hz}, 1 \mathrm{H}, 28-\mathrm{H}), 4.03$ (d, $J=5.0 \mathrm{~Hz}, 1 \mathrm{H}, 28-\mathrm{H}), 3.69$ (t, 1H, 3-H), 3.56-3.42 (m, 3H, 16,21,22-H), 1.92 (t, $J=12.9 \mathrm{~Hz}, 1 \mathrm{H}$, 18-H), $1.90-1.20\left(\mathrm{~m}, 16 \mathrm{H}, \mathrm{CH}_{2}\right), 1.15-0.70$ (s, 18H, $\left.\mathrm{CH}_{3}, 24,25,26,27,29,30-\mathrm{H}\right) .{ }^{13} \mathrm{C}$ NMR $(151 \mathrm{MHz}$, $\left.\mathrm{C}_{2} \mathrm{D}_{6} \mathrm{SO}\right) \delta: 205.44,167.06,162.71,139.96,130.57,130.52,125.41,123.40,117.39,114.51,76.71$, $75.70,74.52,68.35,65.45,54.08,49.51,47.18,46.61,46.11,43.04,41.64,41.47,35.69,33.23,31.67$, $30.77,30.48,29.50,25.57,25.41,23.45,22.55,19.12,15.75,13.99,9.75 . \mathrm{HRMS}_{(\mathrm{ESI}}{ }^{+}$, calcd for $\mathrm{C}_{37} \mathrm{H}_{52} \mathrm{O}_{7} \mathrm{~F}(\mathrm{M}+\mathrm{H})^{+}, 627.4234$; found, 627.4242 .

28-O-m-bromo benzoyl-3 $\beta, 16 \alpha, 21 \beta, 22 \alpha$-O-tetrahydroxy-oleantel-12-Ene-23-aldehyde (S-8). DMAP $(0.12 \mathrm{~g}, 1.0 \mathrm{mmol})$ and intermediate $3 \mathrm{a}(0.67 \mathrm{~g}, 1.0 \mathrm{mmol})$ were dissolved in pyridine $(10 \mathrm{~mL})$ and reacted with 3-bromobenzoyl chloride $(160 \mu \mathrm{L}, 1.2 \mathrm{mmol})$ at $40^{\circ} \mathrm{C}$ for $24 \mathrm{~h}$. The post-processing was the same as that of S-4 and sapogenin derivative $\mathbf{S - 8}$ was obtained as gray powder $(0.5 \mathrm{~g}, 72.71 \%)$. IR (KBr) 3523, 3068, 2928, 2858, 1724, 1594, 1510, 1469, 747, 809, $533 \mathrm{~cm}^{-1} .{ }^{1} \mathrm{H}$ NMR (400 MHz, $\left.\mathrm{C}_{2} \mathrm{D}_{6} \mathrm{SO}\right) \delta: 9.40(\mathrm{~s}, 1 \mathrm{H}, \mathrm{CHO}), 8.05(\mathrm{~s}, 1 \mathrm{H}), 7.94(\mathrm{~d}, J=7.8 \mathrm{~Hz}, 1 \mathrm{H}), 7.84(\mathrm{~d}, J=8.8 \mathrm{~Hz}, 1 \mathrm{H}), 7.50(\mathrm{~m}$, $J=7.9 \mathrm{~Hz}, 1 \mathrm{H}), 6.74(\mathrm{~s}, 1 \mathrm{H}, \mathrm{OH}, 3-\mathrm{H}), 5.61(\mathrm{~s}, 1 \mathrm{H}, \mathrm{OH}, 21-\mathrm{H}), 5.45(\mathrm{~s}, 1 \mathrm{H}, \mathrm{OH}, 22-\mathrm{H}), 5.14(\mathrm{t}, 1 \mathrm{H}$, 12-H), 4.59 (s, 1H, OH, 16-H), 4.33 (d, J=5.1 Hz, 1H, 28-H), 3.96 (d, J=7.0 Hz, 1H, 28-H), 3.57 (t, 1H, 3-H), 3.45 (m, 3H, 16,21,22-H), $1.92(\mathrm{t}, J=9.4 \mathrm{~Hz}, 1 \mathrm{H}, 18-\mathrm{H}), 2.0-1.20\left(\mathrm{~m}, 16 \mathrm{H}, \mathrm{CH}_{2}\right), 1.19$ 
0.80 (s, $\left.18 \mathrm{H}, \mathrm{CH}_{3}, 24,25,26,27,29,30-\mathrm{H}\right) .{ }^{13} \mathrm{C} \mathrm{NMR}\left(151 \mathrm{MHz}, \mathrm{C}_{2} \mathrm{D}_{6} \mathrm{SO}\right) \delta: 205.96,166.39,144.53$, $136.00,132.20,131.89,131.32,128.71,122.32,122.16,77.26,74.68,70.95,67.63,60.20,56.50,47.97$, $46.82,46.65,44.75,43.09,41.68,41.50,38.86,36.89,35.95,33.23,31.75,27.40,24.97,23.49,21.52$, 21.50, 21.21, 19.12, 19.01, 14.54. HRMS $\left(\mathrm{ESI}^{+}\right)$, calcd for $\mathrm{C}_{37} \mathrm{H}_{52} \mathrm{O}_{7} \mathrm{Br}(\mathrm{M}+\mathrm{H})^{+}, 688.4792$; found, 688.4782.

28-O-m-methyl benzoyl-3 $\beta, 16 \alpha, 21 \beta, 22 \alpha$-O-tetrahydroxy-oleantel-12-Ene-23-aldehyde (S-9). DMAP $(0.12 \mathrm{~g}, 1.0 \mathrm{mmol})$ and intermediate $3 \mathrm{a}(0.67 \mathrm{~g}, 1.0 \mathrm{mmol})$ were dissolved in pyridine $(10 \mathrm{~mL})$ and reacted with 3-methylbenzophenyl chloride $(160 \mu \mathrm{L}, 1.2 \mathrm{mmol})$ at $40^{\circ} \mathrm{C}$ for $24 \mathrm{~h}$. The post-processing was the same as that of S-4 and sapogenin derivative $\mathbf{S - 9}$ was obtained as white powder $(0.53 \mathrm{~g}$, 85.09\%). IR (KBr) 3517, 3065, 2938, 2858, 1714, 1595, 1525, 1456, 746, $806 \mathrm{~cm}^{-1} .{ }^{1} \mathrm{H}$ NMR (400 $\left.\mathrm{MHz}, \mathrm{C}_{2} \mathrm{D}_{6} \mathrm{SO}\right) \delta: 9.42(\mathrm{~s}, 1 \mathrm{H}, \mathrm{CHO}), 7.82(\mathrm{~d}, J=8.6 \mathrm{~Hz}, 1 \mathrm{H}), 7.76(\mathrm{~s}, 1 \mathrm{H}), 7.42(\mathrm{~m}, J=8.3 \mathrm{~Hz}, 1 \mathrm{H})$, $7.18(\mathrm{~d}, J=7.8 \mathrm{~Hz}, 1 \mathrm{H}), 6.72(\mathrm{~s}, 1 \mathrm{H}, \mathrm{OH}, 3-\mathrm{H}), 5.31(\mathrm{~s}, 2 \mathrm{H}, \mathrm{OH}, 21,22-\mathrm{H}), 5.04$ (t, $J=4.4 \mathrm{~Hz}, 1 \mathrm{H}$, 12-H), 4.65 (s, 1H, OH, 16-H), 4.47 (d, J=10.4 Hz, 1H, 28-H), 3.99 (d, J=25.1 Hz, 1H, 28-H), $3.82(\mathrm{t}$ 1H, 3-H), 3.42-3.30 (m, 3H,16,21,22-H), 2.36 (s, 3H, $\left.\mathrm{CH}_{3}\right), 2.17$ (t, $\left.J=5.2 \mathrm{~Hz}, 1 \mathrm{H}, 18-\mathrm{H}\right), 1.83-1.23$ $\left(\mathrm{m}, 16 \mathrm{H}, \mathrm{CH}_{2}\right), 1.20-0.70\left(\mathrm{~s}, 18 \mathrm{H}, \mathrm{CH}_{3}, 24,25,26,27,29,30-\mathrm{H}\right) .{ }^{13} \mathrm{C}$ NMR $\left(151 \mathrm{MHz}, \mathrm{C}_{2} \mathrm{D}_{6} \mathrm{SO}\right) \delta$ : $204.61,165.95,142.20,138.30,133.86,130.18,129.99,128.87,126.90,123.09,77.28,74.67,70.68$, $68.16,59.55,55.30,47.05,46.69,46.56,45.43,43.98,41.57,41.52,38.87,36.91,35.70,33.69,33.30$, 27.42, 25.12, 24.97, 23.50, 22.57, 21.28, 21.05, 16.48, 15.77, 14.38. HRMS (ESI $\left.{ }^{+}\right)$, calcd for $\mathrm{C}_{38} \mathrm{H}_{55} \mathrm{O}_{7}$ $(\mathrm{M}+\mathrm{H})^{+}, 623.5902$; found, 623.5913 .

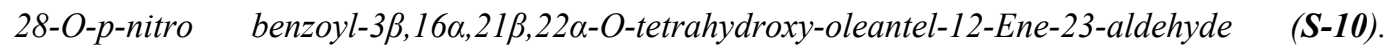
DMAP $(0.12 \mathrm{~g}, 1.0 \mathrm{mmol})$ and intermediate 3a $(0.67 \mathrm{~g}, 1.0 \mathrm{mmol})$ were dissolved in pyridine $(10 \mathrm{~mL})$ and reacted with 4-nitrobenzoyl chloride $(0.22 \mathrm{~g}, 1.2 \mathrm{mmol})$ at $40^{\circ} \mathrm{C}$ for $24 \mathrm{~h}$. The post-processing was the same as that of S-4 and sapogenin derivative S-10 was obtained as light-yellow powder $(0.53 \mathrm{~g}$, 81.07\%). IR (KBr) 3509, 3112, 2928, 2860, 1724, 1604, 1459, 1529, 1350, $840 \mathrm{~cm}^{-1} .{ }^{1} \mathrm{H}$ NMR (400 $\left.\mathrm{MHz}, \mathrm{C}_{2} \mathrm{D}_{6} \mathrm{SO}\right) \delta: 9.43(\mathrm{~s}, 1 \mathrm{H}, \mathrm{CHO}), 8.37(\mathrm{~d}, J=14.1 \mathrm{~Hz}, 2 \mathrm{H}), 8.20(\mathrm{~d}, J=10.3 \mathrm{~Hz}, 2 \mathrm{H}), 6.74(\mathrm{~s}, 1 \mathrm{H}$, OH, 3-H), 5.33 (s, 2H, OH, 21,22-H), 5.05 ( t, $J=4.4$ Hz, 1H, 12-H), 4.70 (s, 1H, OH, 16-H), 4.33 (d, $J=4.6 \mathrm{~Hz}, 1 \mathrm{H}, 28-\mathrm{H}), 4.02(\mathrm{~d}, J=12.2 \mathrm{~Hz}, 1 \mathrm{H}, 28-\mathrm{H}), 3.85(\mathrm{t}, 1 \mathrm{H}, 3-\mathrm{H}), 3.65-3.50(\mathrm{~m}, 3 \mathrm{H}, 16,21$, 22-H), 1.99 (t, $J=7.0 \mathrm{~Hz}, 1 \mathrm{H}, 18-\mathrm{H}), 1.85-1.10\left(\mathrm{~m}, 16 \mathrm{H}, \mathrm{CH}_{2}\right), 1.05-0.70\left(\mathrm{~s}, 18 \mathrm{H}, \mathrm{CH}_{3}, 24,25,26,27\right.$, 29, 30-H). ${ }^{13} \mathrm{C}$ NMR (151 MHz, $\left.\mathrm{C}_{2} \mathrm{D}_{6} \mathrm{SO}\right) \delta: 202.29,164.49,150.80,142.22,131.40,130.08,130.08$, $124.44,124.12,123.93,75.41,70.89,67.97,65.83,62.26,55.29,47.01,46.69,46.60,45.05,43.65$, 
$41.57,41.37,38.87,36.91,34.13,31.77,27.05,25.10,24.96,23.53,22.56,20.63,19.12,16.48,15.84$, 14.39. HRMS $\left(\mathrm{ESI}^{+}\right)$, calcd for $\mathrm{C}_{37} \mathrm{H}_{52} \mathrm{NO}_{9}(\mathrm{M}+\mathrm{H})^{+}$, 654.6572; found, 654.6564.

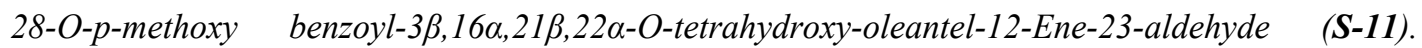
DMAP $(0.12 \mathrm{~g}, 1.0 \mathrm{mmol})$ and intermediate 3a $(0.67 \mathrm{~g}, 1.0 \mathrm{mmol})$ were dissolved in pyridine $(10 \mathrm{~mL})$ and reacted with 4-methoxybenzophenyl chloride $(0.20 \mathrm{~g}, 1.2 \mathrm{mmol})$ at $40^{\circ} \mathrm{C}$ for $24 \mathrm{~h}$. The post-processing was the same as that of S-4 and sapogenin derivative S-11 was obtained as white powder (0.49 g, 76.70\%). IR (KBr) 3492, 3065, 2937, 2858, 1687, 1605, 1510, 1458, 1259, 1028, 746, $806 \mathrm{~cm}^{-1} .{ }^{1} \mathrm{H}$ NMR (400 MHz, $\left.\mathrm{C}_{2} \mathrm{D}_{6} \mathrm{SO}\right) \delta: 9.25(\mathrm{~s}, 1 \mathrm{H}, 23-\mathrm{H}), 7.89(\mathrm{~d}, J=8.9 \mathrm{~Hz}, 2 \mathrm{H}), 7.02(\mathrm{~d}, J=8.9$ Hz, 2H), 6.80 (s, 1H, OH, 3-H), 5.32 (s, 2H, OH, 21,22-H), 5.03 (t, J=4.4 Hz, 1H, 12-H), 4.65 (s, $1 \mathrm{H}$, OH, 16-H), 4.34 (d, $J=5.1 \mathrm{~Hz}, 1 \mathrm{H}, 28-\mathrm{H}), 4.15$ (d, $J=5.8 \mathrm{~Hz}, 1 \mathrm{H}, 28-\mathrm{H}), 3.89$ (t, 1H, 3-H), 3.83 (s, $\left.3 \mathrm{H}, \mathrm{CH}_{3}\right), 3.50-3.39(\mathrm{~m}, 3 \mathrm{H}, 16,21,22-\mathrm{H}), 2.09(\mathrm{t}, J=3.6 \mathrm{~Hz}, 1 \mathrm{H}, 18-\mathrm{H}), 1.90-1.20\left(\mathrm{~m}, 16 \mathrm{H}, \mathrm{CH}_{2}\right)$, $1.10-0.80\left(\mathrm{~s}, 18 \mathrm{H}, \mathrm{CH}_{3}, 24,25,26,27,29,30-\mathrm{H}\right) .{ }^{13} \mathrm{C} \mathrm{NMR}\left(151 \mathrm{MHz}, \mathrm{C}_{2} \mathrm{D}_{6} \mathrm{SO}\right) \delta: 207.69,167.44$, $165.61,143.32,131.79,131.41,123.45,122.66,114.55,114.24,77.29,75.42,71.66,67.95,64.28$, $55.89,55.86,47.03,46.80,46.65,46.47,43.94,41.67,41.16,38.87,36.91,35.69,33.71,31.76,27.48$, 25.33, 25.18, 23.49, 22.56, 19.01, 16.49, 14.39, 9.35. HRMS $\left(\mathrm{ESI}^{+}\right)$, calcd for $\mathrm{C}_{38} \mathrm{H}_{55} \mathrm{O}_{8}(\mathrm{M}+\mathrm{H})^{+}$, 639.6842; found, 639.6849 .

28-O-(2-thiophenoformyl)-3 $\beta, 16 \alpha, 21 \beta, 22 \alpha$-O-tetrahydroxy-oleantel-12-Ene-23-aldehyde

$(S-12)$.

DMAP (0.12 g, $1.0 \mathrm{mmol})$ and intermediate 3a $(0.67 \mathrm{~g}, 1.0 \mathrm{mmol})$ were dissolved in pyridine $(10 \mathrm{~mL})$ and reacted with 2-thiophenoformyl chloride $(130 \mu \mathrm{L}, 1.2 \mathrm{mmol})$ at $40^{\circ} \mathrm{C}$ for $24 \mathrm{~h}$. The post-processing was the same as that of S-4 and sapogenin derivative S-12 was obtained as yellow powder $(0.53 \mathrm{~g}$, 86.20\%). IR (KBr) 3500, 3100, 2931, 2858, 1702, 1643, 1525, $744 \mathrm{~cm}^{-1} .{ }^{1} \mathrm{H}$ NMR (400 MHz, $\left.\mathrm{C}_{2} \mathrm{D}_{6} \mathrm{SO}\right)$ $\delta: 9.37$ (s, 1H, CHO), 7.92 (d, $J=15.7 \mathrm{~Hz}, 1 \mathrm{H}), 7.80$ (d, $J=14.5 \mathrm{~Hz}, 1 \mathrm{H}), 7.12$ (t, $J=4.3 \mathrm{~Hz}, 1 \mathrm{H})$, $6.76(\mathrm{~s}, 1 \mathrm{H}, \mathrm{OH}, 3-\mathrm{H}), 5.30$ (s, 2H, OH, 21,22-H), 5.19 (t, 1H, 12-H), 4.59 (s, 1H, OH, 16-H), $4.22(\mathrm{~d}$, $J=6.5 \mathrm{~Hz}, 1 \mathrm{H}, 28-\mathrm{H}), 4.03(\mathrm{~d}, J=7.1 \mathrm{~Hz}, 1 \mathrm{H}, 28-\mathrm{H}), 3.44(\mathrm{~m}, 4 \mathrm{H}, 3,16,21,22-\mathrm{H}), 1.98(\mathrm{t}, J=7.6 \mathrm{~Hz}$, 1H, 18-H), $1.90-1.20\left(\mathrm{~m}, 16 \mathrm{H}, \mathrm{CH}_{2}\right), 1.10-0.80\left(\mathrm{~s}, 18 \mathrm{H}, \mathrm{CH}_{3}, 24,25,26,27,29,30-\mathrm{H}\right) .{ }^{13} \mathrm{C}$ NMR $(151$ $\left.\mathrm{MHz}, \mathrm{C}_{2} \mathrm{D}_{6} \mathrm{SO}\right) \delta: 208.35,170.74,143.96,131.93,130.08,129.10,128.08,122.02,77.29,74.46,71.64$, $67.61,60.22,55.32,47.41,46.98,46.65,45.18,44.06,41.65,41.27,38.88,36.93,34.13,33.78,31.77$, 27.25, 25.44, 24.97, 23.45, 22.57, 21.49, 19.19, 16.88, 14.40. HRMS (ESI ${ }^{+}$), calcd for $\mathrm{C}_{35} \mathrm{H}_{51} \mathrm{O}_{7} \mathrm{~S}$ $(\mathrm{M}+\mathrm{H})^{+}, 615.4317$; found, 615.4326. 
DMAP $(0.12 \mathrm{~g}, 1.0 \mathrm{mmol})$ and intermediate 3a $(0.67 \mathrm{~g}, 1.0 \mathrm{mmol})$ were dissolved in pyridine $(10 \mathrm{~mL})$ and reacted with 2 -furan formyl chloride $(120 \mu \mathrm{L}, 1.2 \mathrm{mmol})$ at $40^{\circ} \mathrm{C}$ for $24 \mathrm{~h}$. The post-processing was the same as that of S-4 and sapogenin derivative $\mathbf{S - 1 3}$ was obtained as white powder $(0.49 \mathrm{~g}, 81.84 \%)$. IR (KBr) 3520, 3132, 2930, 2858, 1713, 1642, $1390 \mathrm{~cm}^{-1} .{ }^{1} \mathrm{H}$ NMR (400 MHz, $\left.\mathrm{C}_{2} \mathrm{D}_{6} \mathrm{SO}\right) \delta: 9.36$ (s, $1 \mathrm{H}$, CHO), $7.94(\mathrm{~d}, J=17.3 \mathrm{~Hz}, 1 \mathrm{H}), 7.27$ (d, $J=3.5 \mathrm{~Hz}, 1 \mathrm{H}), 6.67\left(\mathrm{dd}, J_{1}=3.4 \mathrm{~Hz}, J_{2}=1.7 \mathrm{~Hz}, 1 \mathrm{H}\right), 6.59$ (s, 1H, OH, 3-H), 5.31 (s, 2H, OH, 21,22-H), 5.20 (t, J = 5.2 Hz, 1H, 12-H), 4.94 (s, 1H, OH, 16-H), 4.35 (d, $J=10.1 \mathrm{~Hz}, 1 \mathrm{H}, 28-\mathrm{H}), 3.95$ (d, $J=6.9 \mathrm{~Hz}, 1 \mathrm{H}, 28-\mathrm{H}), 3.64$ (t, 1H, 3-H), 3.57 (m, 3H, 16,21, 22-H), 1.98 (t, $J=6.4 \mathrm{~Hz}, 1 \mathrm{H}, 18-\mathrm{H}), 1.85-1.20\left(\mathrm{~m}, 16 \mathrm{H}, \mathrm{CH}_{2}\right), 1.10-0.88\left(\mathrm{~s}, 18 \mathrm{H}, \mathrm{CH}_{3}, 24,25,26,27\right.$, 29,30-H). ${ }^{13} \mathrm{C}$ NMR (151 MHz, $\left.\mathrm{C}_{2} \mathrm{D}_{6} \mathrm{SO}\right) \delta: 205.45,158.01,148.06,144.79,143.95,123.44,118.32$, $112.56,74.25,73.91,68.00,67.67,67.64,54.48,47.00,46.63,46.12,46.11,42.95,41.59,41.46,38.06$, $36.83,35.67,33.26,31.76,27.46,24.99,23.43,22.56,20.46,17.15,15.77,9.72$. HRMS $_{\left(\mathrm{ESI}^{+}\right) \text {, calcd }}$ for $\mathrm{C}_{35} \mathrm{H}_{50} \mathrm{O}_{8}(\mathrm{M}+\mathrm{H})^{+}$, 599.4232; found, 599.4248.

28-O-(2-hydroxybenzoyl)-3 $\beta, 16 \alpha, 21 \beta, 22 \alpha$-O-tetrahydroxy-oleantel-12-Ene-23-aldehyde

(S-14). Salicylic acid $(0.17 \mathrm{~g}, 1.2 \mathrm{mmol}), \mathrm{EDC} \cdot \mathrm{HCl}(0.19 \mathrm{~g}, 1.0 \mathrm{mmol})$ and DMAP $(0.12 \mathrm{~g}, 1.0 \mathrm{mmol})$ were dissolved in pyridine $(10 \mathrm{~mL})$, activated in ice water bath for $2 \mathrm{~h}$ and reacted with intermediate $3 \mathbf{a}$ at $40^{\circ} \mathrm{C}$ for $24 \mathrm{~h}$. The post-processing was the same as that of S-4 and sapogenin derivative $\mathbf{S - 1 4}$ was obtained as white powder (0.39 g, 62.22\%). IR (KBr) 3389, 2930; 2858, 1719, 1673, 1600, 1557, 1457, $757 \mathrm{~cm}^{-1} .{ }^{1} \mathrm{H}$ NMR (400 MHz, $\left.\mathrm{C}_{2} \mathrm{D}_{6} \mathrm{SO}\right) \delta: 10.45$ (s, 1H, OH), 9.25 (s, 1H, CHO), 7.78 (d, J=8.0 Hz, 1H), 7.52 (t, $J=7.7 \mathrm{~Hz}, 1 \mathrm{H}), 6.96(\mathrm{~m}, J=8.0 \mathrm{~Hz}, 2 \mathrm{H}), 6.73(\mathrm{~s}, 1 \mathrm{H}, \mathrm{OH}, 3-\mathrm{H}), 5.56(\mathrm{~s}, 2 \mathrm{H}, \mathrm{OH}$, 21,22-H), 5.32 (t, $J=4.7 \mathrm{~Hz}, 1 \mathrm{H}, 12-\mathrm{H}), 4.64$ (s, 1H, OH, 16-H), 4.32 (d, J=4.4 Hz, 1H, 28-H), 3.93 (d, $J=7.4 \mathrm{~Hz}, 1 \mathrm{H}, 28-\mathrm{H}), 3.70-3.60$ (m, 4H, 3,16,21,22-H), 1.99 (t, $J=10.4 \mathrm{~Hz}, 1 \mathrm{H}, 18-\mathrm{H}), 1.90-1.20$ $\left(\mathrm{m}, 16 \mathrm{H}, \mathrm{CH}_{2}\right), 1.10-0.70\left(\mathrm{~s}, 18 \mathrm{H}, \mathrm{CH}_{3}, 24,25,26,27,29,30-\mathrm{H}\right) .{ }^{13} \mathrm{C} \mathrm{NMR}\left(151 \mathrm{MHz}, \mathrm{C}_{2} \mathrm{D}_{6} \mathrm{SO}\right) \delta$ : $207.50,168.37,160.19,143.17,136.05,130.43,122.03,119.85,118.22,113.75,77.29,70.05,69.03$ $67.37,62.96,55.58,47.99,47.00,46.62,45.36,43.58,41.66,41.55,38.88,36.92,34.13,33.68,31.77$, 27.05, 25.80, 24.96, 23.48, 22.56, 16.49, 14.39, 9.35. HRMS $\left(\mathrm{ESI}^{+}\right)$, calcd for $\mathrm{C}_{37} \mathrm{H}_{53} \mathrm{O}_{8}(\mathrm{M}+\mathrm{H})^{+}$, 625.3642; found, 625.3645 .

28-O-(5-bromo-2-hydroxybenzoyl)-3 $\beta, 16 \alpha, 21 \beta, 22 \alpha$-O-tetrahydroxy-oleantel-12-Ene-23-aldehyde (S-15). 5-Bromosalicylic acid (0.26 g, $1.2 \mathrm{mmol}), \mathrm{EDC} \cdot \mathrm{HCl}(0.19 \mathrm{~g}, 1.0 \mathrm{mmol})$ and DMAP $(0.12 \mathrm{~g}$, $1.0 \mathrm{mmol})$ were dissolved in pyridine $(10 \mathrm{~mL})$, activated in ice water bath for $2 \mathrm{~h}$ and reacted with intermediate $3 \mathbf{a}$ at $40^{\circ} \mathrm{C}$ for $24 \mathrm{~h}$. The post-processing was the same as that of $\mathbf{S - 4}$ and sapogenin 
derivative S-15 was obtained as white powder (0.42 g, 59.52\%). IR (KBr) 3389, 2930, 2858, 1719 , 1673, 1600, 1557, 1457, 829, 883, $532 \mathrm{~cm}^{-1} .{ }^{1} \mathrm{H}$ NMR (400 MHz, $\left.\mathrm{C}_{2} \mathrm{D}_{6} \mathrm{SO}\right) \delta: 10.49$ (s, 1H, OH), 9.25 (s, 1H, 23-H), $7.86(\mathrm{~s}, 1 \mathrm{H}), 7.67$ (d, $J=5.7 \mathrm{~Hz}, 1 \mathrm{H}), 6.95(\mathrm{~d}, J=8.4 \mathrm{~Hz}, 1 \mathrm{H}), 6.73(\mathrm{~s}, 1 \mathrm{H}, \mathrm{OH}, 3-\mathrm{H})$, $5.56(\mathrm{~s}, 2 \mathrm{H}, \mathrm{OH}, 21,22-\mathrm{H}), 5.23(\mathrm{t}, J=6.7 \mathrm{~Hz}, 1 \mathrm{H}, 12-\mathrm{H}), 4.62(\mathrm{~s}, 1 \mathrm{H}, \mathrm{OH}, 16-\mathrm{H}), 4.19(\mathrm{~d}, J=6.4 \mathrm{~Hz}$, 1H, 28-H), 3.91 (d, $J=11.0 \mathrm{~Hz}, 1 \mathrm{H}, 28-\mathrm{H}), 3.70-3.60(\mathrm{~m}, 4 \mathrm{H}, 3,16,21,22-\mathrm{H}), 1.87$ (t, $J=5.3 \mathrm{~Hz}, 1 \mathrm{H}$, 18-H), 1.85-1.10 (m, 16H, $\left.\mathrm{CH}_{2}\right), 1.10-0.70$ (s, 18H, $\left.\mathrm{CH}_{3}, 24,25,26,27,29,30-\mathrm{H}\right) .{ }^{13} \mathrm{C} \mathrm{NMR}(151 \mathrm{MHz}$, $\left.\mathrm{C}_{2} \mathrm{D}_{6} \mathrm{SO}\right) \delta: 207.51,166.63,157.09,144.43,138.19,132.72,122.79,120.79,120.44,110.51,75.83$, $74.70,70.92,70.03,67.22,55.58,47.99,47.00,46.61,45.33,43.53,41.75,41.60,38.29,36.91,35.69$, $33.66,31.57,29.50,27.26,25.80,23.48,19.06,16.87,15.92,9.35$. HRMS $\left(\mathrm{ESI}^{+}\right)$, calcd for $\mathrm{C}_{37} \mathrm{H}_{52} \mathrm{O}_{8} \mathrm{Br}(\mathrm{M}+\mathrm{H})^{+}, 704.2767$; found, 704.2760.

28-O-(2-mercapto-4-methyl-5-thiazolyl)-3 $\beta, 16 \alpha, 21 \beta, 22 \alpha$-O-tetrahydroxy-oleantel-12-Ene-23-

aldehyde (S-16). 2-Mercapto-4-methyl-5-thiazolylacetic acid (0.23 g, $1.2 \mathrm{mmol}), \mathrm{EDC} \cdot \mathrm{HCl}(0.19 \mathrm{~g}, 1.0$ mmol) and DMAP $(0.12 \mathrm{~g}, 1.0 \mathrm{mmol})$ were dissolved in pyridine $(10 \mathrm{~mL})$, activated in ice water bath for $2 \mathrm{~h}$ and reacted with intermediate $3 \mathbf{a}$ at $40^{\circ} \mathrm{C}$ for $24 \mathrm{~h}$. The post-processing was the same as that of S-4 and sapogenin derivative S-16 was obtained as yellow powder (0.44 g, 65.09\%). IR (KBr) 3354, 2931, 2858, 2597, 1714, $1636 \mathrm{~cm}^{-1} .{ }^{1} \mathrm{H}$ NMR (400 MHz, $\left.\mathrm{C}_{2} \mathrm{D}_{6} \mathrm{SO}\right) \delta: 12.92$ (s, 1H, SH), 9.25 (s, $1 \mathrm{H}$, 23-H), 7.69 (s, 2H), $6.74(\mathrm{~s}, 1 \mathrm{H}, \mathrm{OH}, 3-\mathrm{H}), 5.56(\mathrm{~s}, 2 \mathrm{H}, \mathrm{OH}, 21,22-\mathrm{H}), 5.18(\mathrm{t}, J=3.6 \mathrm{~Hz}, 1 \mathrm{H}, 12-\mathrm{H})$, $4.63(\mathrm{~s}, 1 \mathrm{H}, \mathrm{OH}, 16-\mathrm{H}), 4.22(\mathrm{~d}, J=12.0 \mathrm{~Hz}, 1 \mathrm{H}, 28-\mathrm{H}), 3.99(\mathrm{~d}, J=5.8 \mathrm{~Hz}, 1 \mathrm{H}, 28-\mathrm{H}), 3.70-3.50$ (m, 4H, 3,16,21,22-H), 2.20 (t, $J=6.4 \mathrm{~Hz}, 1 \mathrm{H}, 18-\mathrm{H}), 2.0\left(\mathrm{~s}, 3 \mathrm{H}, \mathrm{CH}_{3}\right), 1.80-1.20\left(\mathrm{~m}, 16 \mathrm{H}, \mathrm{CH}_{2}\right), 1.0-0.70$ (s, 18H, $\left.\mathrm{CH}_{3}, 24,25,26,27,29,30-\mathrm{H}\right) .{ }^{13} \mathrm{C}$ NMR (151 MHz, $\left.\mathrm{C}_{2} \mathrm{D}_{6} \mathrm{SO}\right) \delta: 207.55,162.80,157.10,153.36$, $144.03,131.96,121.84,77.29,71.50,70.92,67.60,65.48,55.60,47.99,47.42,46.81,45.18,44.06$, $41.74,41.23,38.88,36.93,35.71,34.00,32.03,30.74,28.68,27.32,25.80,24.93,23.43,20.70,16.84$, 15.84, 14.01, 9.34. HRMS (ESI+ $)$, calcd for $\mathrm{C}_{36} \mathrm{H}_{54} \mathrm{NO}_{7} \mathrm{~S}_{2}(\mathrm{M}+\mathrm{H})^{+}, 676.6276$; found, 676.6274 .

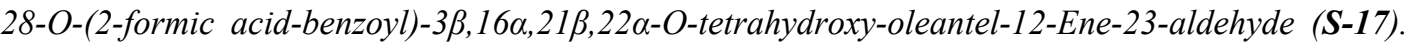
DMAP (0.12 g, $1.0 \mathrm{mmol})$ and intermediate 3a $(0.67 \mathrm{~g}, 1.0 \mathrm{mmol})$ were dissolved in DMSO $(10 \mathrm{~mL})$ and reacted with phthalic anhydride $(0.18 \mathrm{~g}, 1.2 \mathrm{mmol})$ at $40^{\circ} \mathrm{C}$ for $24 \mathrm{~h}$. The post-processing was the same as that of S-4 and sapogenin derivative S-17 was obtained as white powder $(0.54 \mathrm{~g}, 82.72 \%)$. IR (KBr) 3448, 3063, 2932, 2863, 1715, 1650, 1601, 1505, 1454, 1170, $747 \mathrm{~cm}^{-1} .{ }^{1} \mathrm{H}$ NMR (400 MHz, $\left.\mathrm{C}_{2} \mathrm{D}_{6} \mathrm{SO}\right) \delta: 12.48(\mathrm{~s}, 1 \mathrm{H}, \mathrm{COOH}), 9.38(\mathrm{~s}, 1 \mathrm{H}, \mathrm{CHO}), 8.09-8.00(\mathrm{~m}, 2 \mathrm{H}), 7.68-7.57(\mathrm{~m}, 2 \mathrm{H}), 6.44(\mathrm{~s}$, 1H, OH, 3-H), 5.53 (s, 1H, OH, 21-H), 5.47 (s, 1H, OH, 22-H), 5.17 (t, 1H, 12-H), 4.65 (s, 1H, OH, 
16-H), $4.30(\mathrm{~d}, J=11.6 \mathrm{~Hz}, 1 \mathrm{H}, 28-\mathrm{H}), 3.97(\mathrm{~d}, J=6.8 \mathrm{~Hz}, 1 \mathrm{H}, 28-\mathrm{H}), 3.70-3.55(\mathrm{~m}, 4 \mathrm{H}$, 3,16,21,22-H), 1.99 (t, $J=13.5 \mathrm{~Hz}, 1 \mathrm{H}, 18-\mathrm{H}), 1.90-1.20\left(\mathrm{~m}, 16 \mathrm{H}, \mathrm{CH}_{2}\right), 1.0-0.70\left(\mathrm{~s}, 18 \mathrm{H}, \mathrm{CH}_{3}\right.$, 24,25,26,27,29,30-H). ${ }^{13} \mathrm{C}$ NMR (151 MHz, $\left.\mathrm{C}_{2} \mathrm{D}_{6} \mathrm{SO}\right) \delta: 207.42,176.36,164.35,161.18,159.65$, $156.64,147.27,136.12,129.95,122.14,77.30,71.58,70.92,67.62,60.32,55.31,47.42,46.65,45.18$, $44.06,43.04,41.65,41.24,38.87,36.92,33.79,32.82,31.59,28.67,27.26,25.45,25.18,23.44,22.56$, 16.88, 16.49, 15.83. HRMS (ESI $\left.{ }^{+}\right)$, calcd for $\mathrm{C}_{38} \mathrm{H}_{53} \mathrm{O}_{9}(\mathrm{M}+\mathrm{H})^{+}, 653.4211$; found, 653.4210 .

28-O-(3,4,5,6-tetrachloro-2-formicacid-benzoyl)-3 $\beta, 16 \alpha, 21 \beta, 22 \alpha$-O-tetrahydroxy-oleantel-12-Ene-2 3-aldehyde (S-18). DMAP (0.12 g, $1.0 \mathrm{mmol})$ and intermediate 3a (0.67 g, $1.0 \mathrm{mmol})$ were dissolved in DMSO $(10 \mathrm{~mL})$ and reacted with tetrachloro phthalic anhydride $(0.34 \mathrm{~g}, 1.2 \mathrm{mmol})$ at $40^{\circ} \mathrm{C}$ for $24 \mathrm{~h}$. The post-processing was the same as that of $\mathbf{S - 4}$ and sapogenin derivative $\mathbf{S - 1 8}$ was obtained as white powder (0.6 g, 82.72\%). IR (KBr) 3336, 2933, 2858, 1717, 1653, 1600, 1506, 1454, 1316, 1172, 763 $\mathrm{cm}^{-1} .{ }^{1} \mathrm{H}$ NMR (400 MHz, $\left.\mathrm{C}_{2} \mathrm{D}_{6} \mathrm{SO}\right) \delta: 12.48(\mathrm{~s}, 1 \mathrm{H}, \mathrm{COOH}), 9.38(\mathrm{~s}, 1 \mathrm{H}, \mathrm{CHO}), 6.45(\mathrm{~s}, 1 \mathrm{H}, \mathrm{OH}, 3-\mathrm{H})$, $6.20(\mathrm{~s}, 2 \mathrm{H}, \mathrm{OH}, 21,22-\mathrm{H}), 5.19$ (t, 1H, 12-H), 4.56 (s, 1H, OH, 16-H), 4.29 (d, 1H, 28-H), 3.93 (d, 1H, 28-H), 3.70-3.55 (m, 4H, 3,16,21,22-H), $2.0(\mathrm{t}, J=13.1 \mathrm{~Hz}, 1 \mathrm{H}, 18-\mathrm{H}), 1.95-1.20\left(\mathrm{~m}, 16 \mathrm{H}, \mathrm{CH}_{2}\right)$, $1.0-0.70$ (s, $\left.18 \mathrm{H}, \mathrm{CH}_{3}, 24,25,26,27,29,30-\mathrm{H}\right) .{ }^{13} \mathrm{C} \mathrm{NMR}\left(151 \mathrm{MHz}, \mathrm{C}_{2} \mathrm{D}_{6} \mathrm{SO}\right) \delta: 207.53,164.35,161.18$, $144.00,136.12,129.95,122.14,122.05,121.83,77.30,71.59,70.92,67.60,67.38,55.59,47.41,46.82$, $46.64,46.48,44.06,41.73,41.24,38.87,36.92,35.70,33.99,32.82,27.32,26.47,25.45,23.44,20.70$, 18.49, 15.95, 9.35. HRMS (ESI $\left.{ }^{+}\right)$, calcd for $\mathrm{C}_{38} \mathrm{H}_{49} \mathrm{O}_{9} \mathrm{Cl}_{4}(\mathrm{M}+\mathrm{H})^{+}, 790.8623$; found, 790.8629 .

28-O-(4-acetylamino-benzenesulfonyl)-3 $\beta, 16 \alpha, 21 \beta, 22 \alpha$-O-tetrahydroxy-oleantel-12-Ene-23-

aldehyde (S-19). DMAP (0.12 g, $1.0 \mathrm{mmol})$ and intermediate $\mathbf{3 a}(0.67 \mathrm{~g}, 1.0 \mathrm{mmol})$ were dissolved in pyridine $(10 \mathrm{~mL})$ and reacted with paracetamylbenzene sulfonyl chloride $(0.28 \mathrm{~g}, 1.2 \mathrm{mmol})$ at $40^{\circ} \mathrm{C}$ for $24 \mathrm{~h}$. The post-processing was the same as that of S-4 and sapogenin derivative $\mathbf{S - 1 9}$ was obtained as white powder (0.55 g, 83.35\%). IR (KBr) 3419, 3112, 2935, 2928, 1719, 1640, 1597, 1458, 842, 783 $\mathrm{cm}^{-1} .{ }^{1} \mathrm{H}$ NMR (400 MHz, $\left.\mathrm{C}_{2} \mathrm{D}_{6} \mathrm{SO}\right) \delta: 10.50(\mathrm{~s}, 1 \mathrm{H}, \mathrm{NH}), 9.25(\mathrm{~s}, 1 \mathrm{H}, 23-\mathrm{H}), 7.86(\mathrm{~d}, J=5.7 \mathrm{~Hz}, 2 \mathrm{H})$, 7.69 (d, J=13.3 Hz, 2H), 6.73 (s, 1H, OH, 3-H), 6.39 (s, 2H, OH, 21,22-H), 5.18 (t, 1H, 12-H), 4.62 (s, 1H, OH, 16-H), $4.21(\mathrm{~d}, J=9.8 \mathrm{~Hz}, 1 \mathrm{H}, 28-\mathrm{H}), 3.92(\mathrm{~d}, J=5.5 \mathrm{~Hz}, 1 \mathrm{H}, 28-\mathrm{H}), 3.80-3.55(\mathrm{~m}, 4 \mathrm{H}$, 3,16,21,22-H), $2.10\left(\mathrm{~s}, 3 \mathrm{H}, \mathrm{CH}_{3}\right), 1.99(\mathrm{t}, J=12.0 \mathrm{~Hz}, 1 \mathrm{H}, 18-\mathrm{H}), 1.90-1.20\left(\mathrm{~m}, 16 \mathrm{H}, \mathrm{CH}_{2}\right), 1.0-0.70$ (s, $\left.18 \mathrm{H}, \mathrm{CH}_{3}, 24,25,26,27,29,30-\mathrm{H}\right) .{ }^{13} \mathrm{C} \mathrm{NMR}\left(151 \mathrm{MHz}, \mathrm{C}_{2} \mathrm{D}_{6} \mathrm{SO}\right) \delta: 207.51,167.40,146.96,144.02$, 143.64, 129.11, 122.08, 119.27, 77.30, 71.53, 70.81, 67.31, 65.47, 55.59, 47.62, 47.42, 46.64, 45.18, 
$42.35,41.74,41.24,38.88,36.63,35.80,35.71,32.04,28.68,27.32,25.46,24.69,23.44,19.22,16.83$, 15.96, 9.35. HRMS $\left(\mathrm{ESI}^{+}\right)$, calcd for $\mathrm{C}_{38} \mathrm{H}_{56} \mathrm{NO}_{9} \mathrm{~S}(\mathrm{M}+\mathrm{H})^{+}, 702.5692$; found, 702.5702 .

${ }^{1} \mathrm{H}$ NMR and ${ }^{13} \mathrm{C}$ NMR Spectrum for sapogenin $\left(\mathrm{C}_{2} \mathrm{D}_{6} \mathrm{SO}\right.$ as solvent $)$

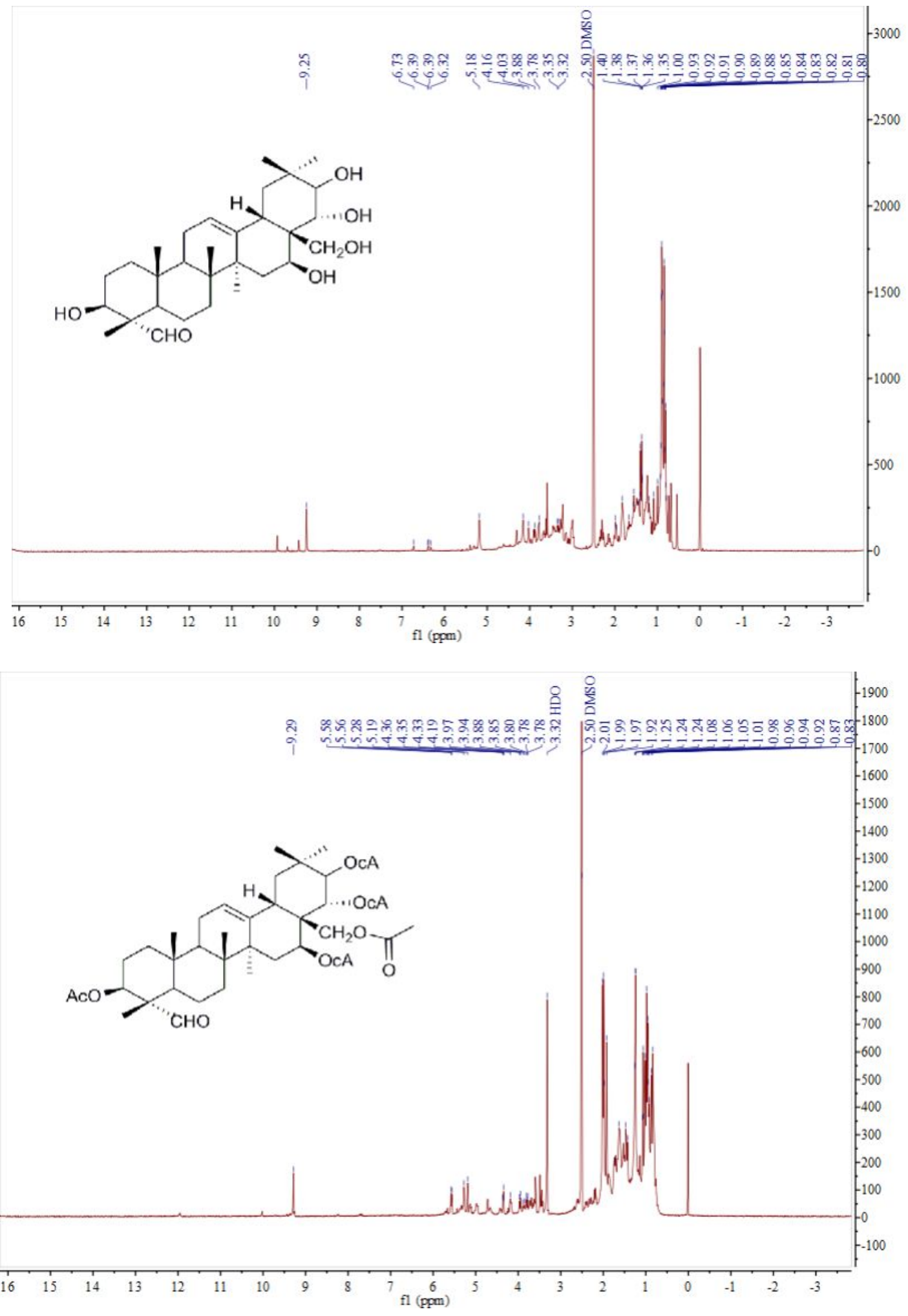


${ }^{1} \mathrm{H}$ NMR and ${ }^{13} \mathrm{C}$ NMR Spectrum for derivative $\mathrm{S}-(1-19)\left(\mathrm{C}_{2} \mathrm{D}_{6} \mathrm{SO}\right.$ as solvent)

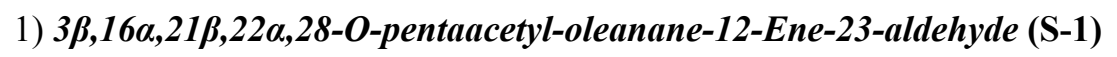
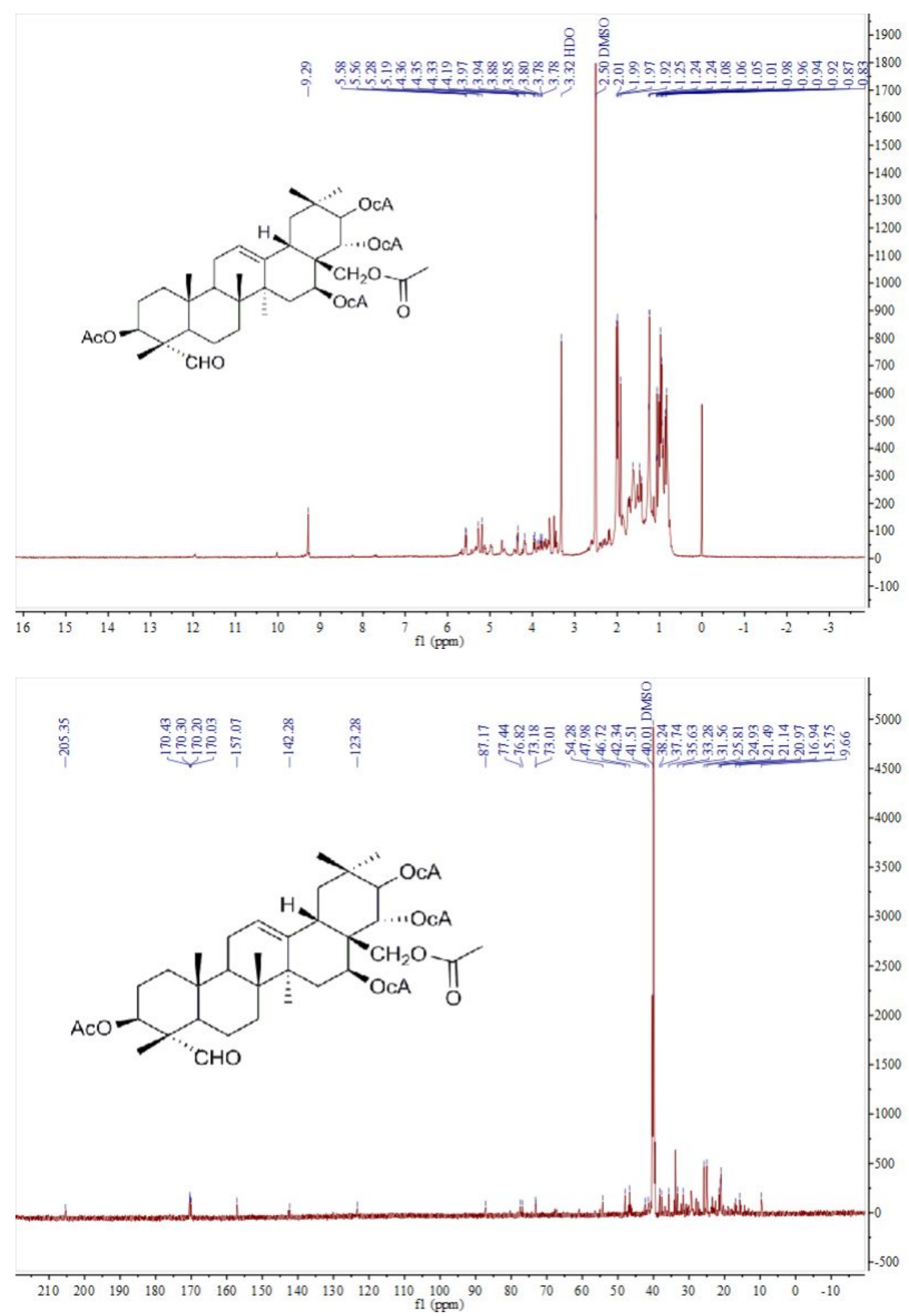


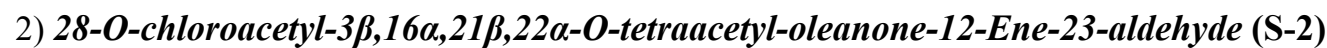
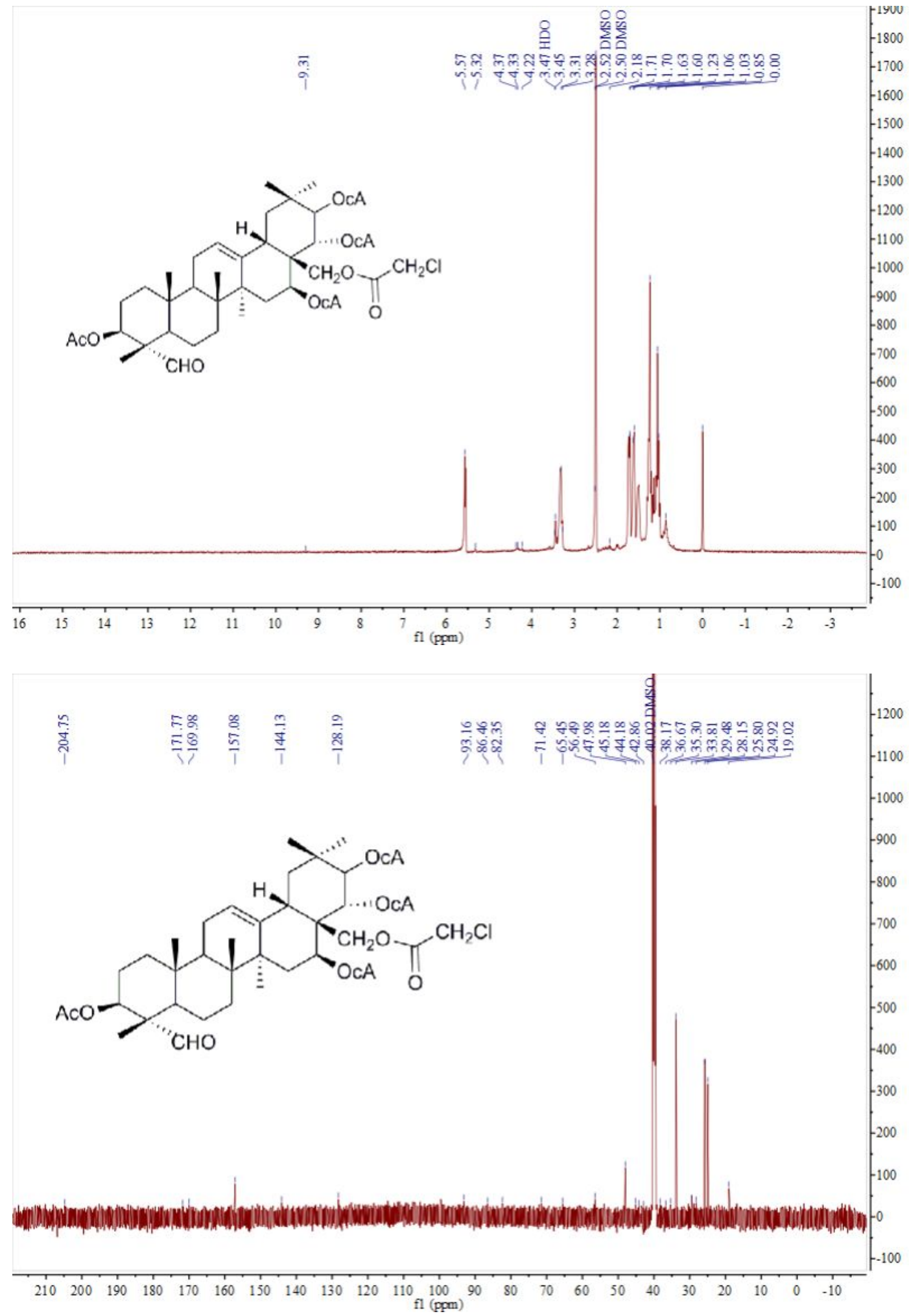


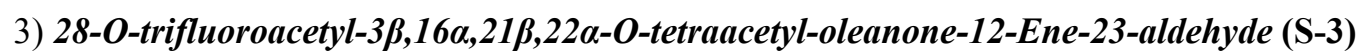
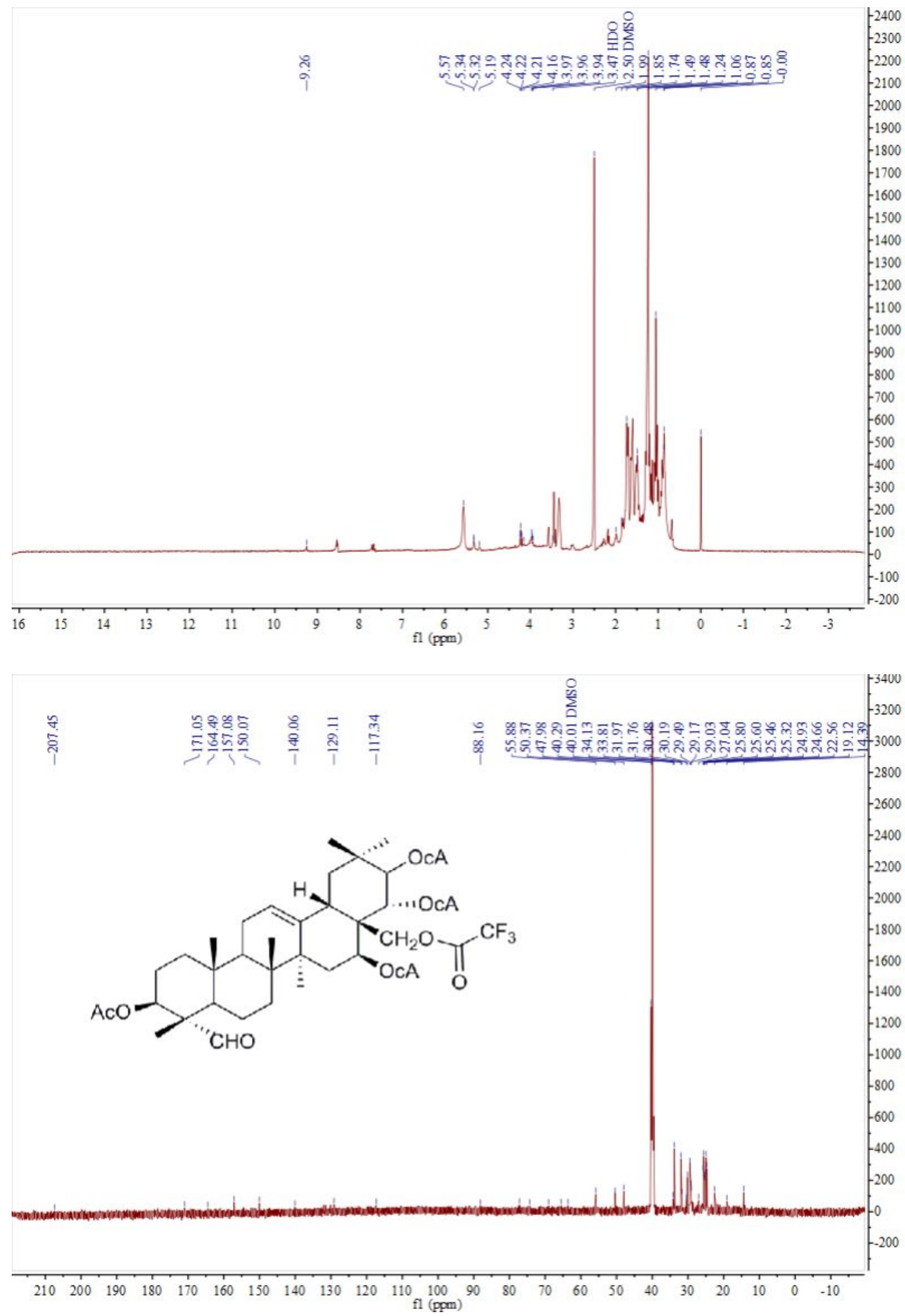


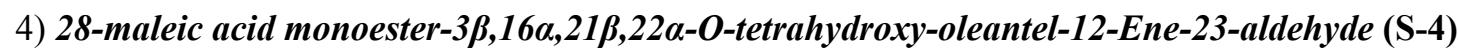

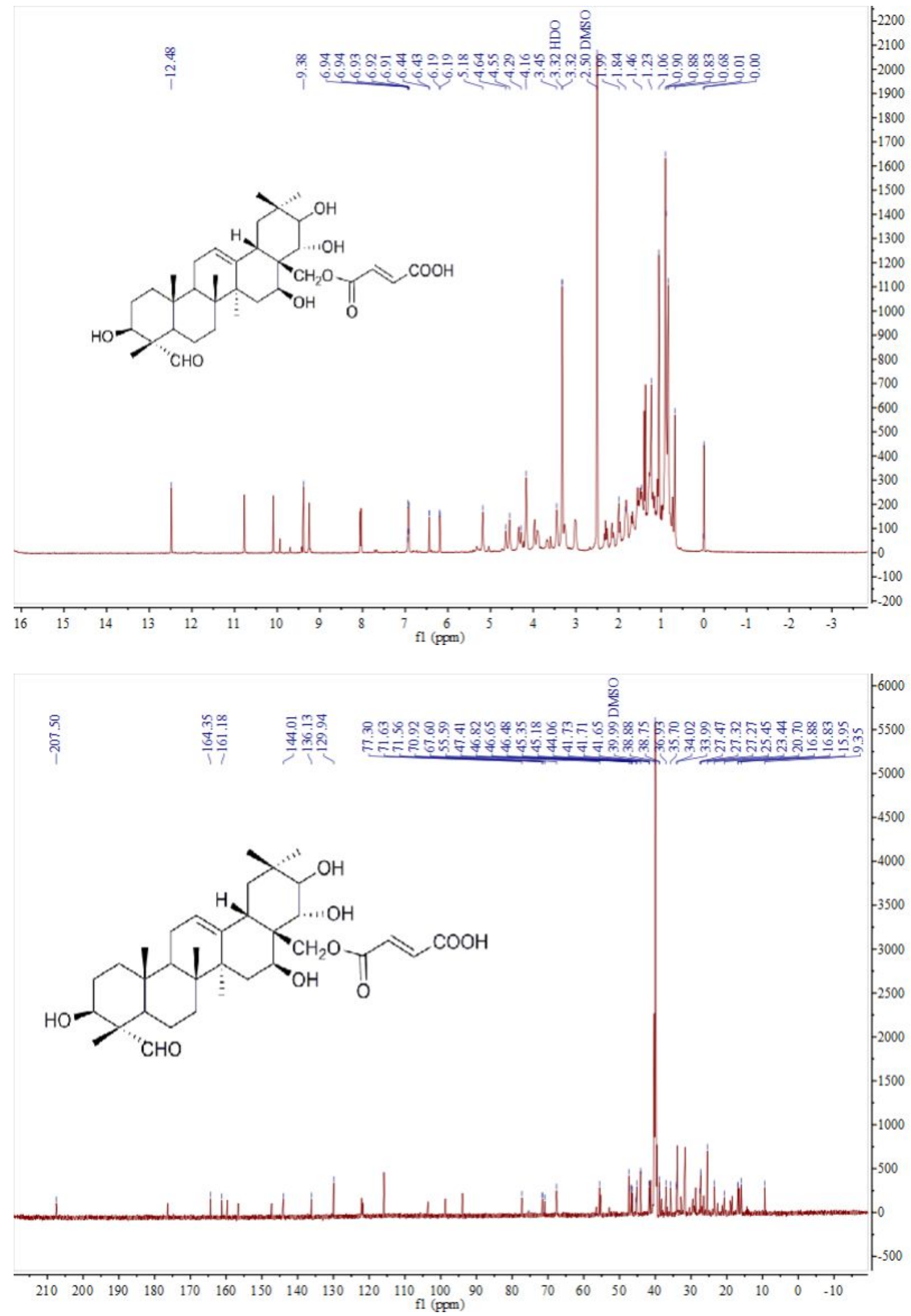




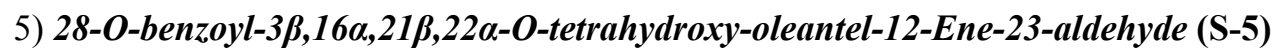
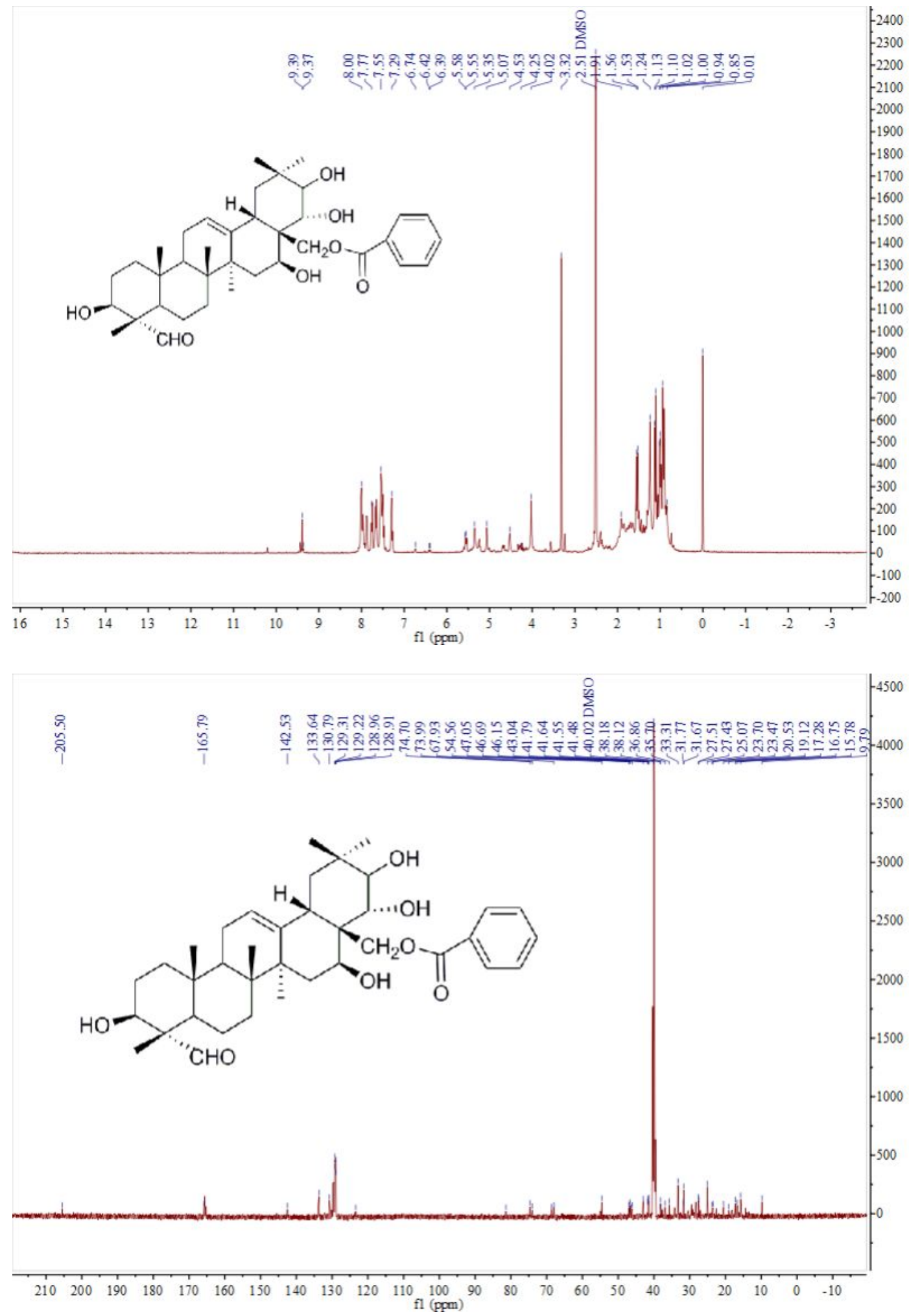


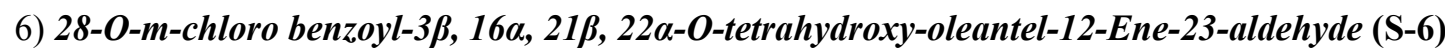
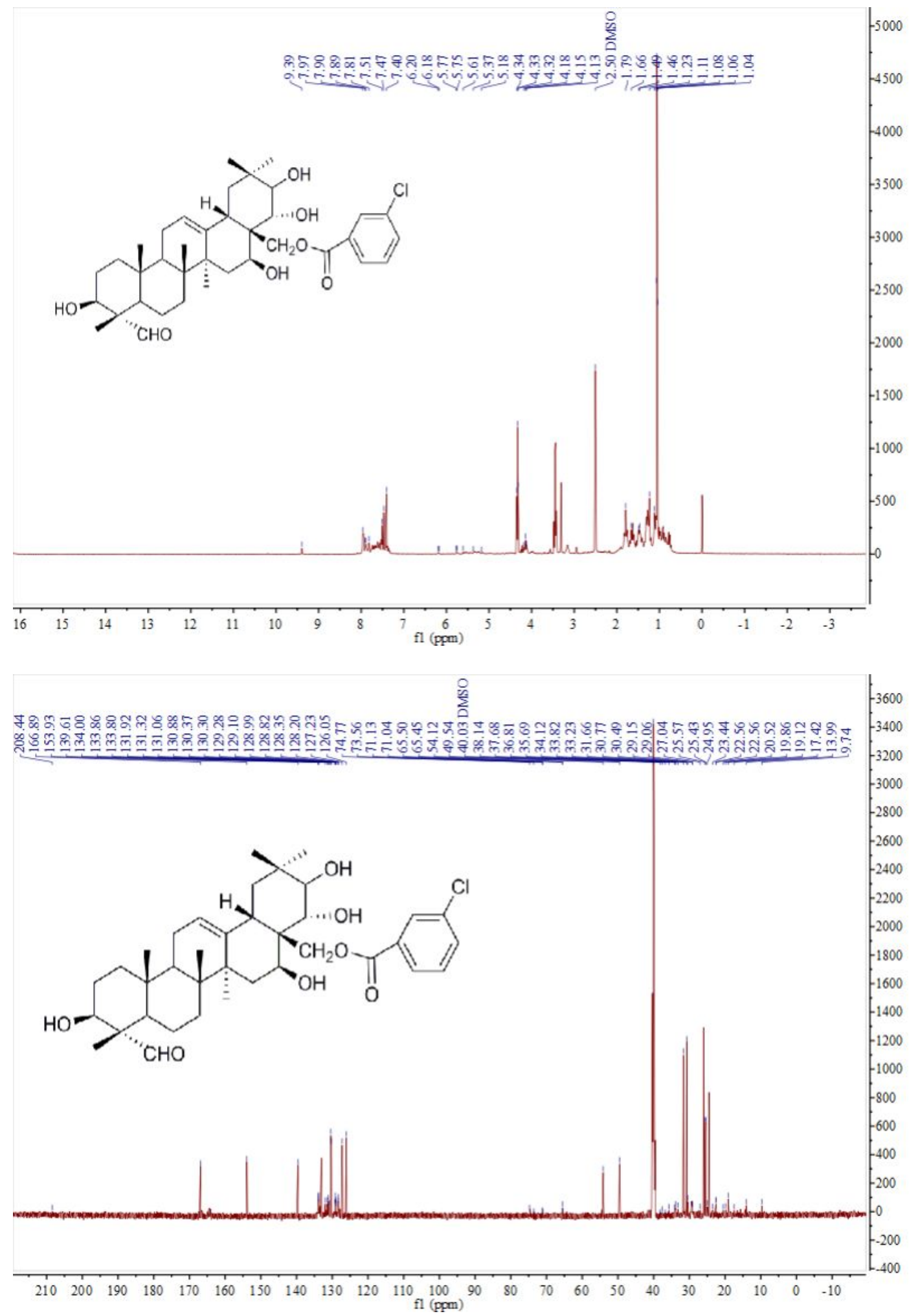


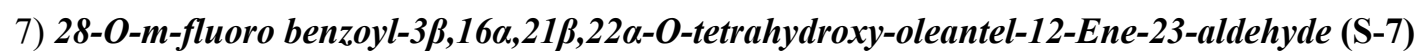

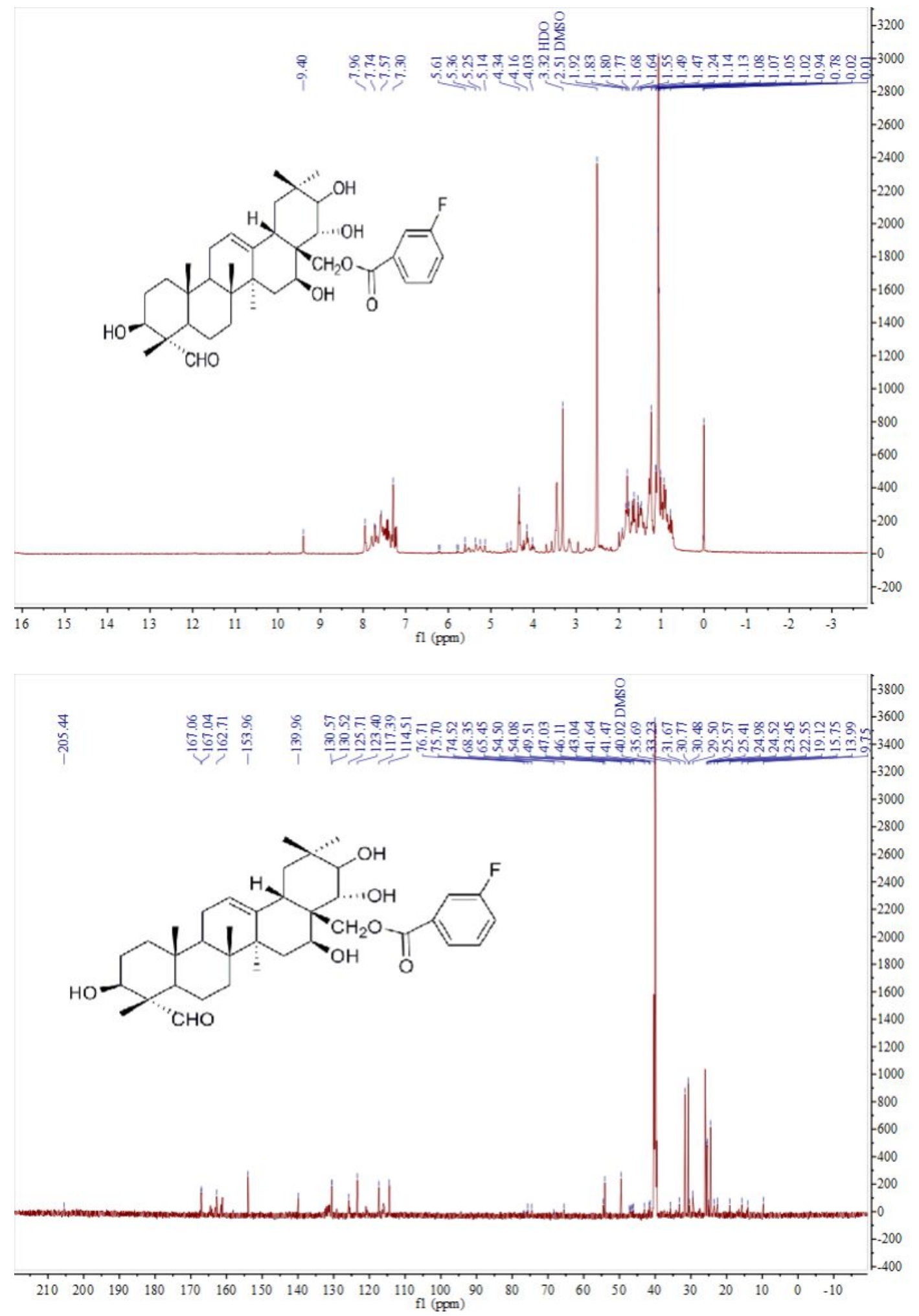




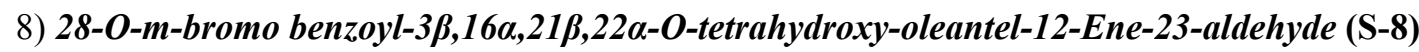
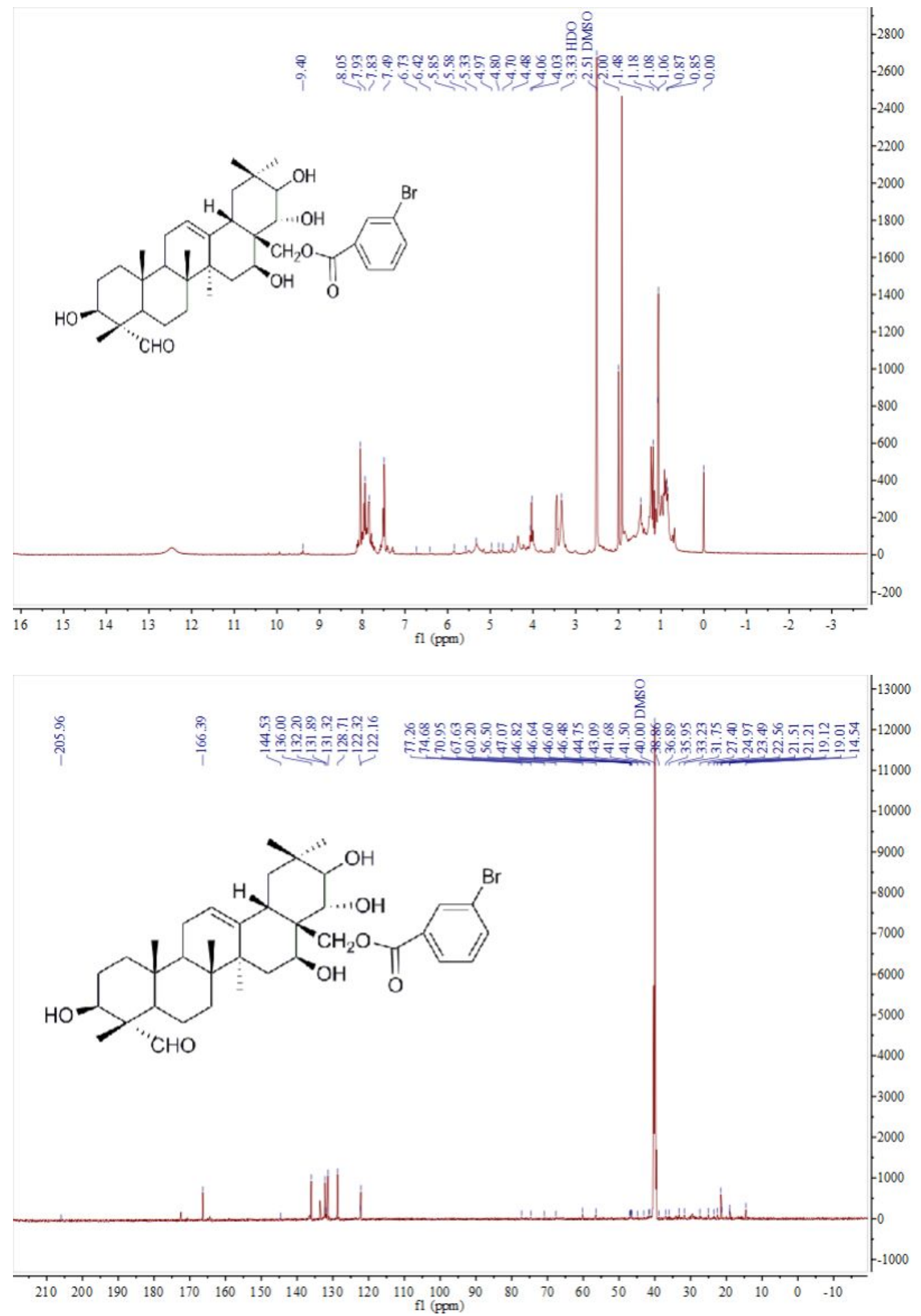


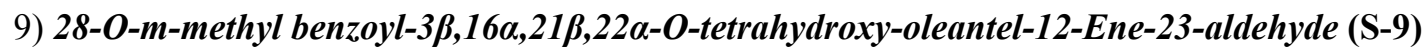
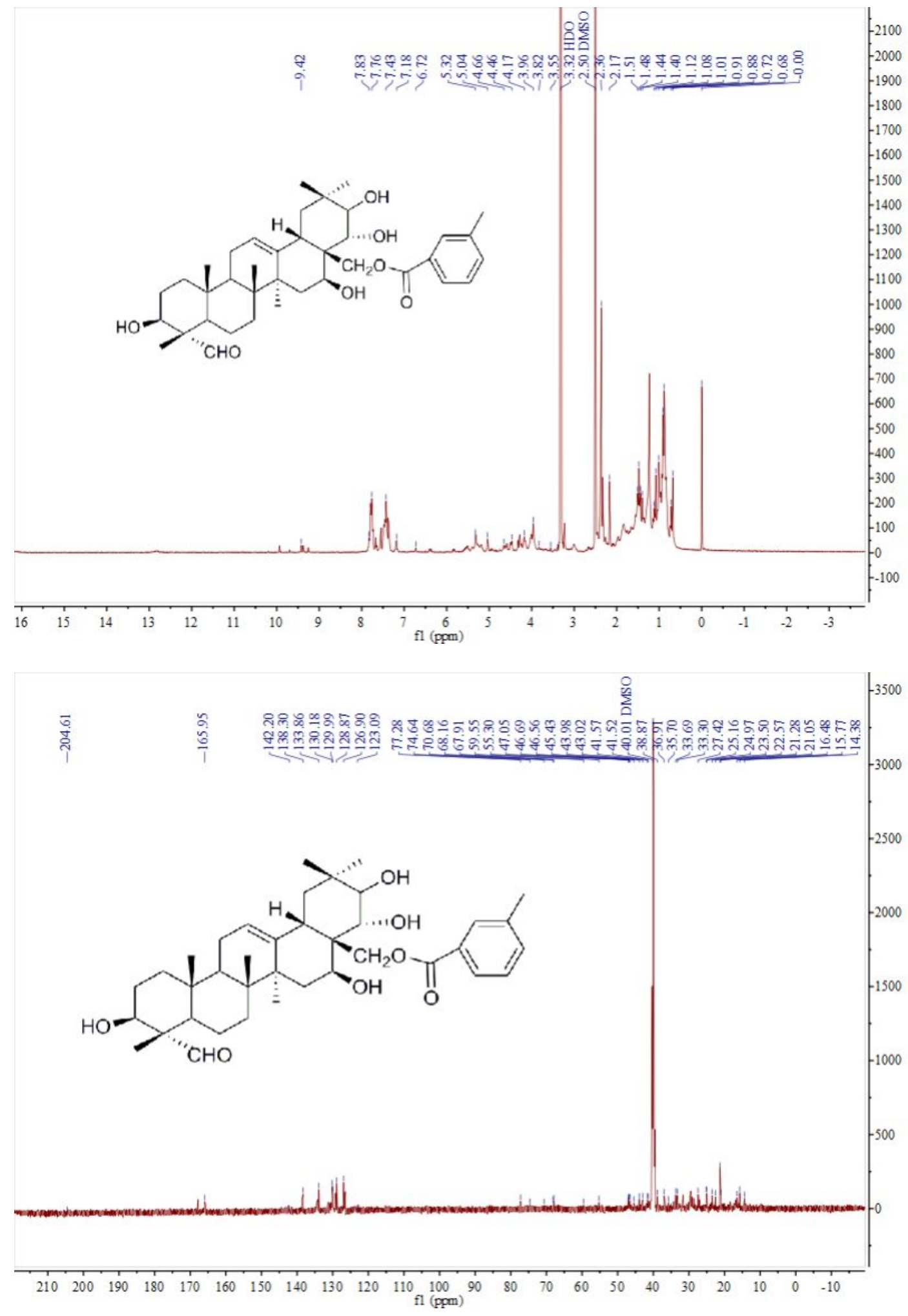


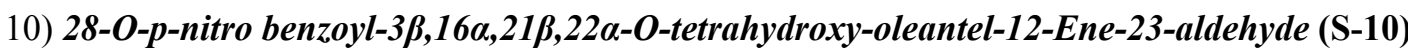

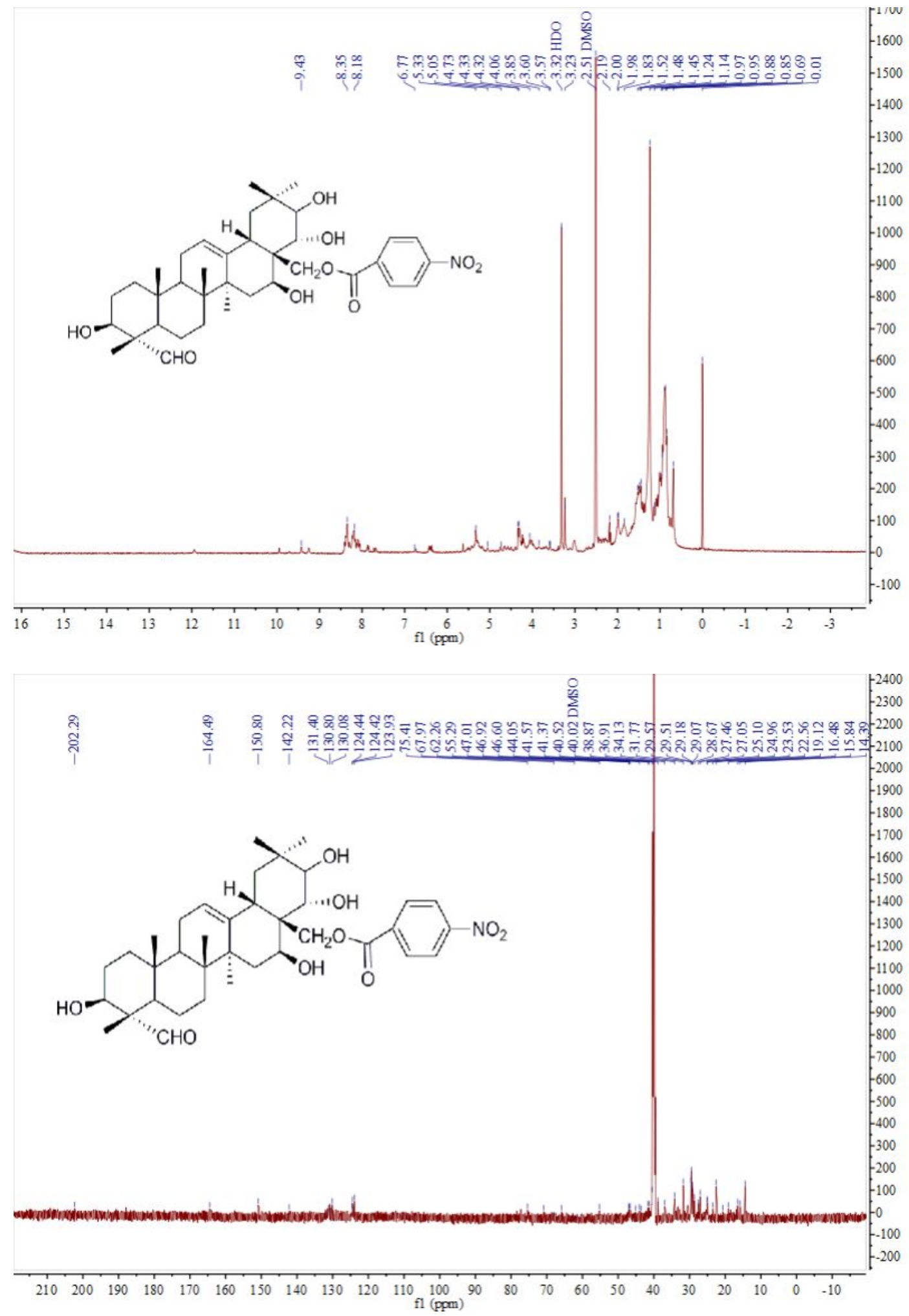




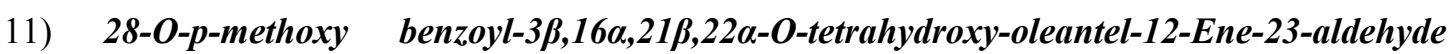
(S-11)
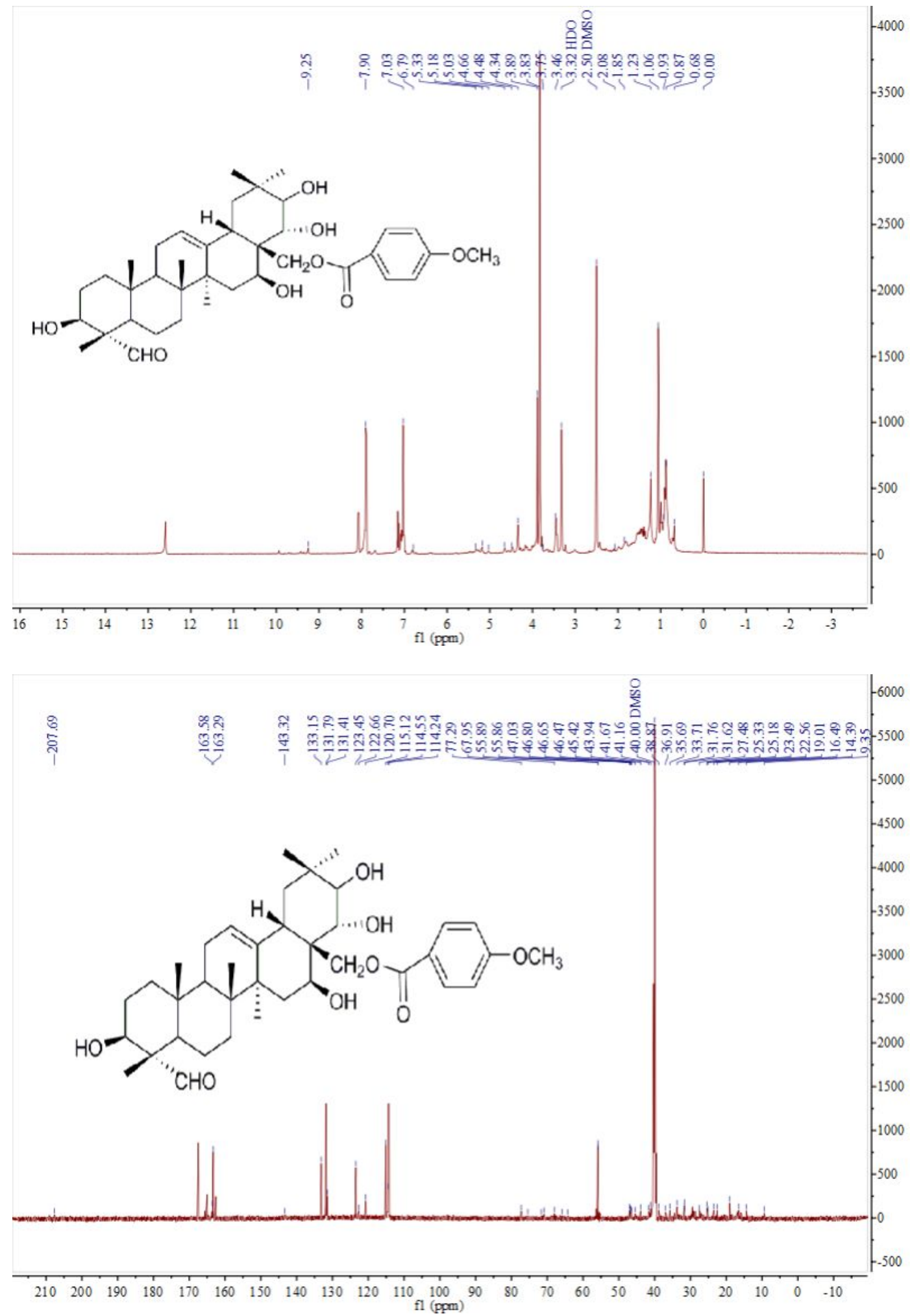


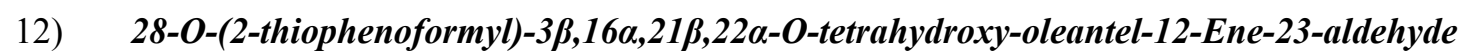
(S-12)

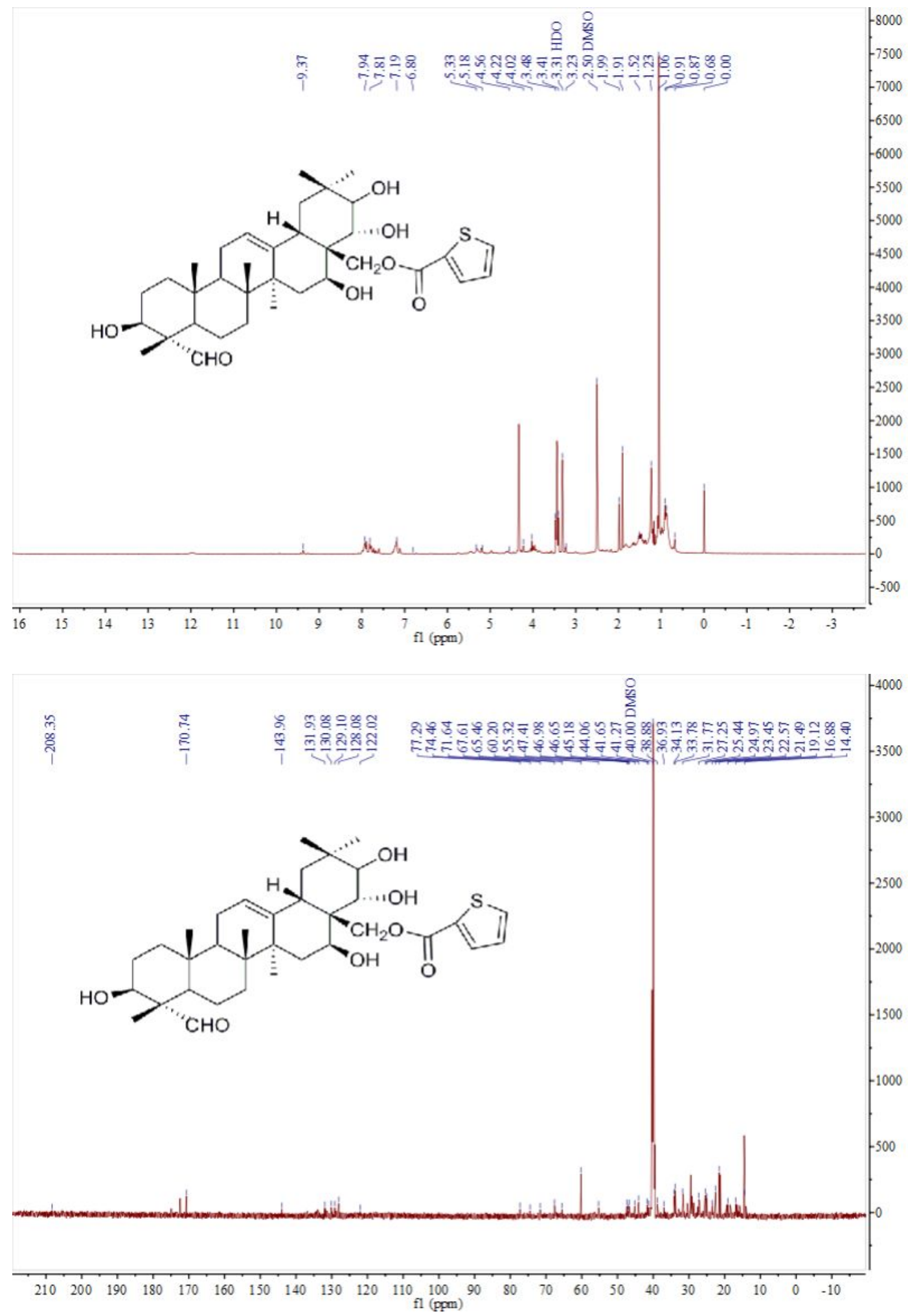




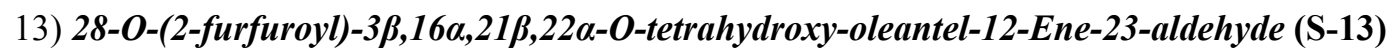
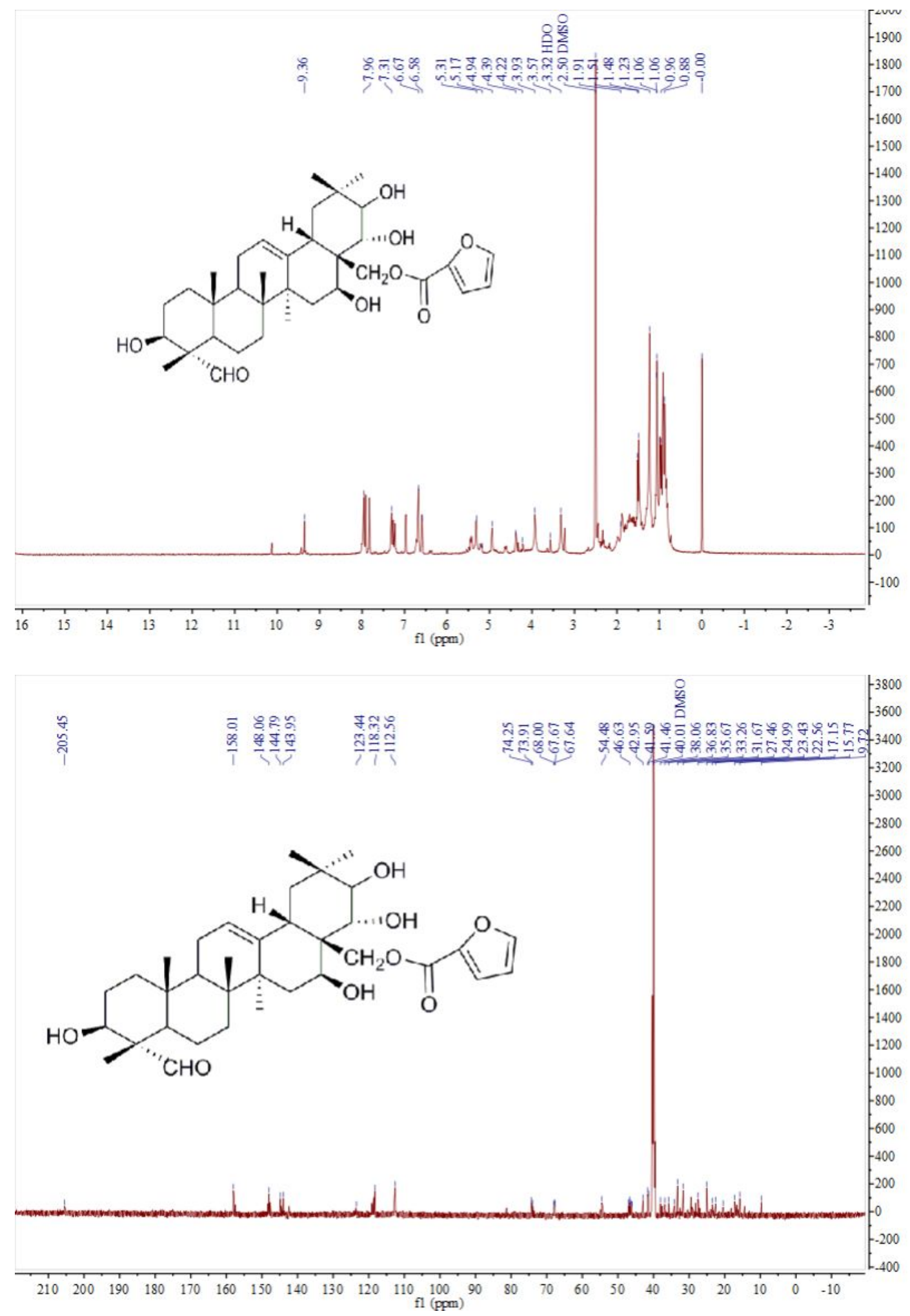


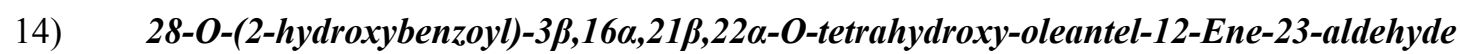
(S-14)

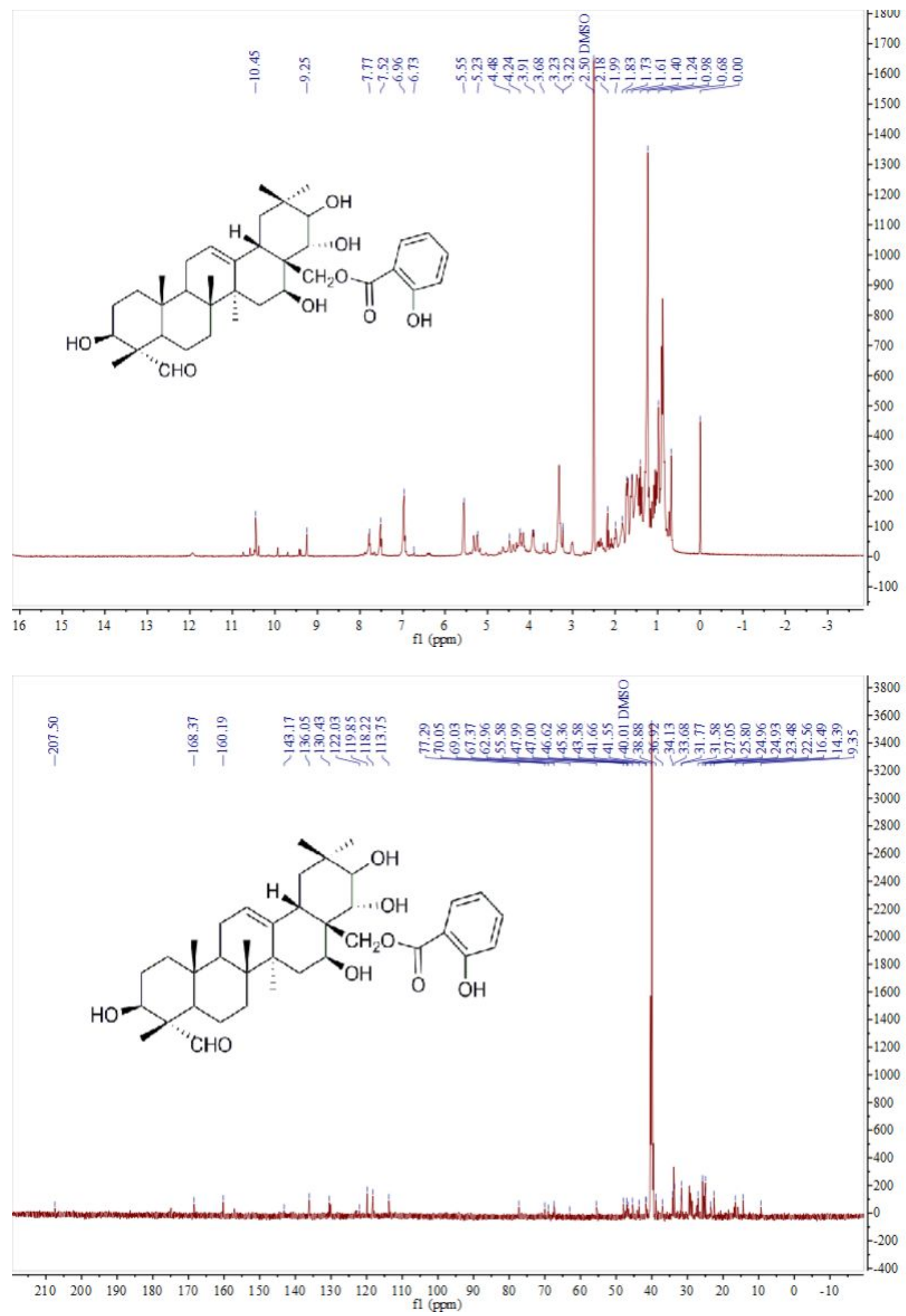




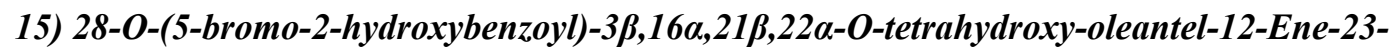
aldehyde (S-15)

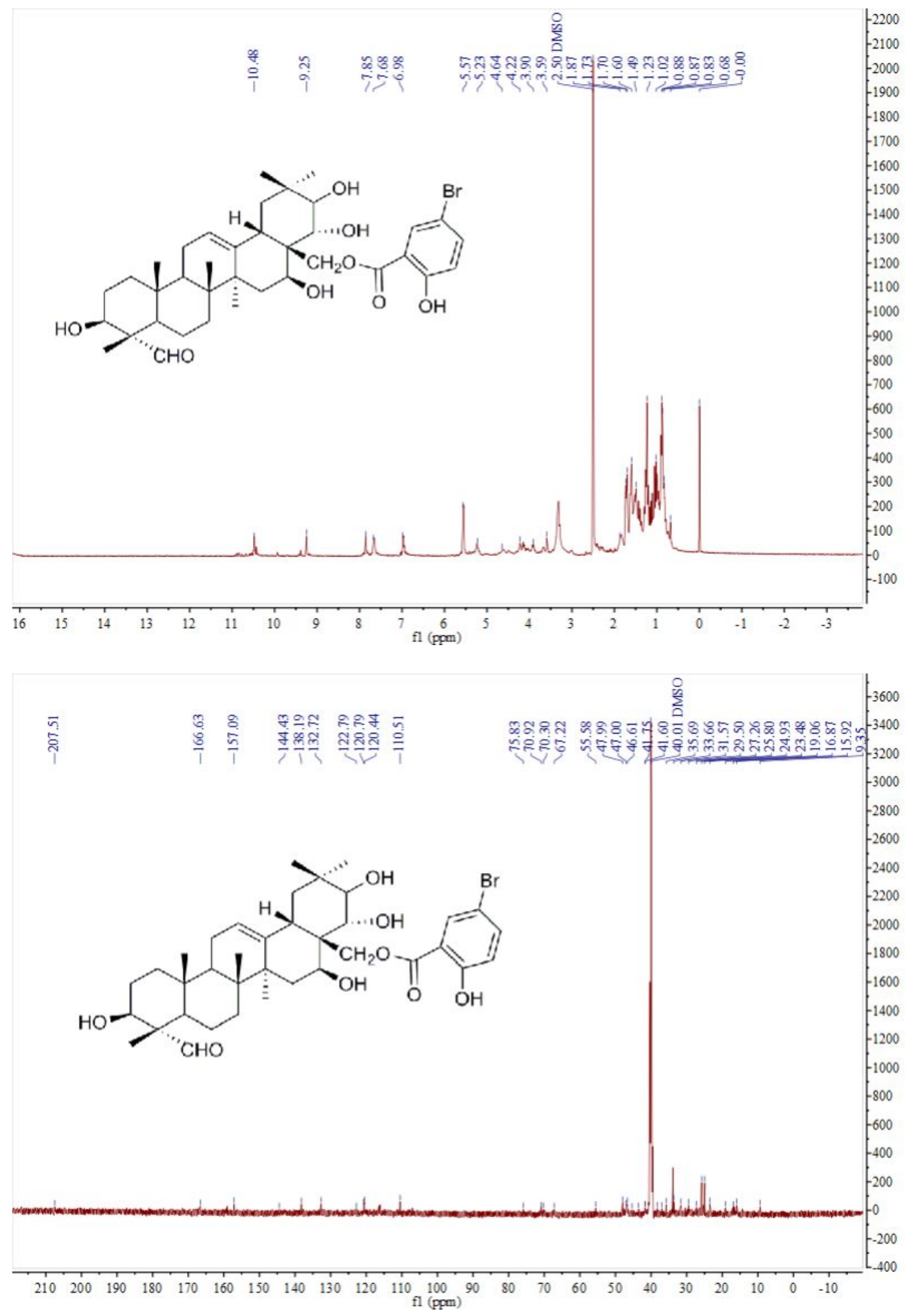




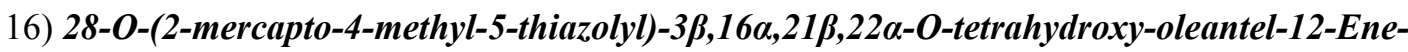
23-aldehyde (S-16)

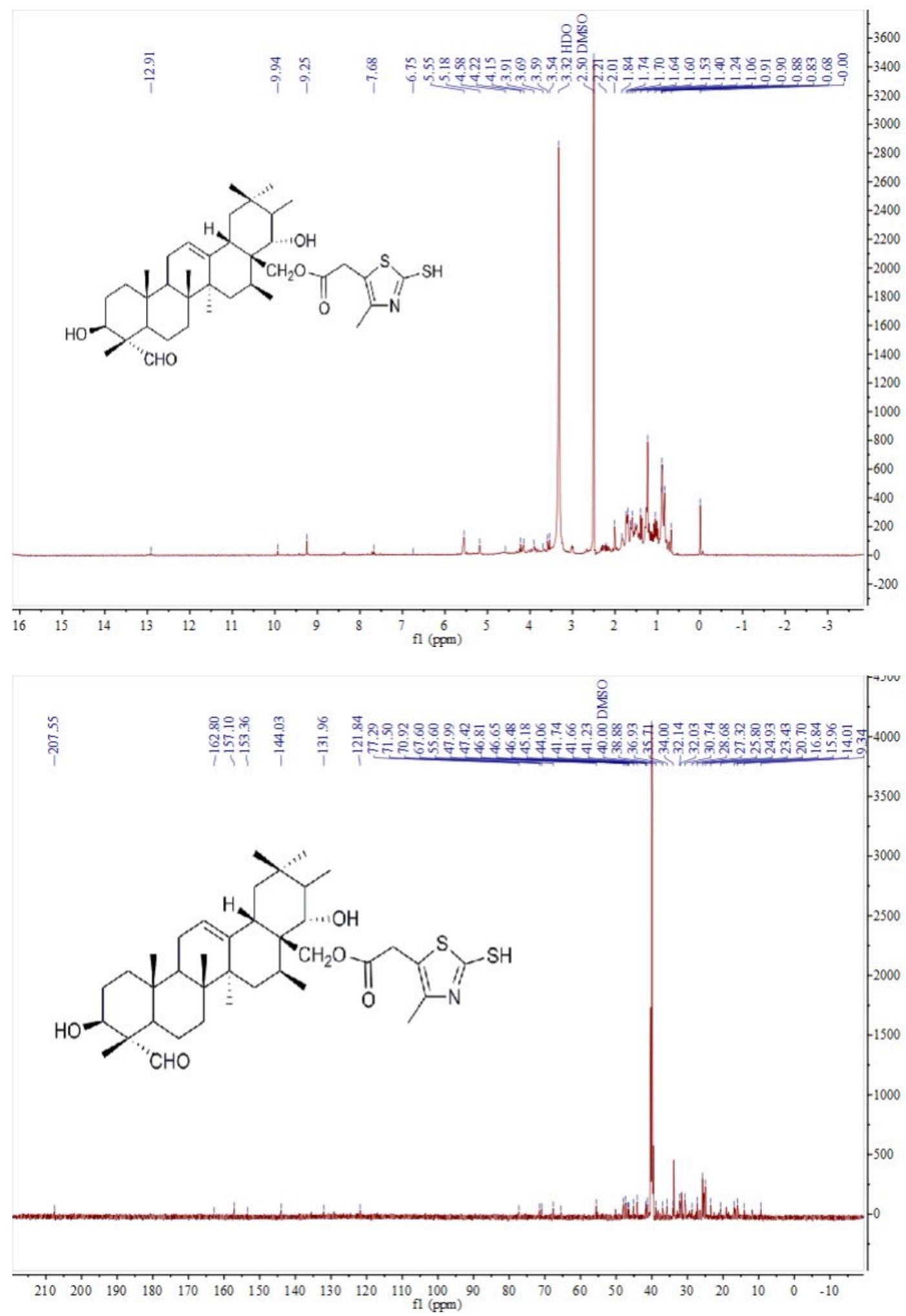




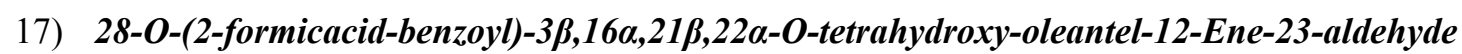
(S-17)
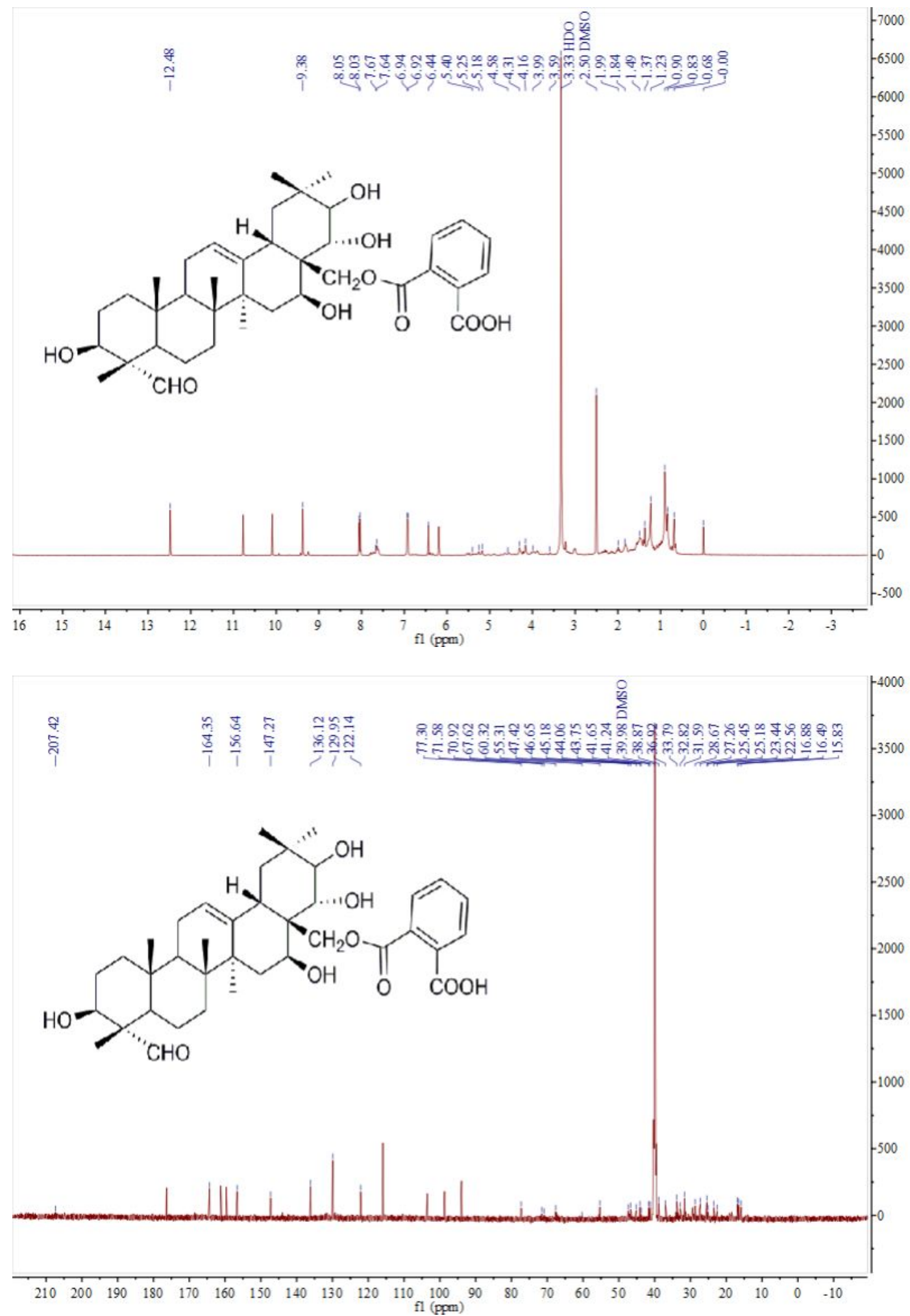


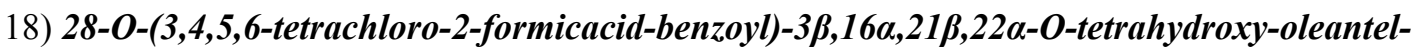
12-Ene-23-aldehyde (S-18)

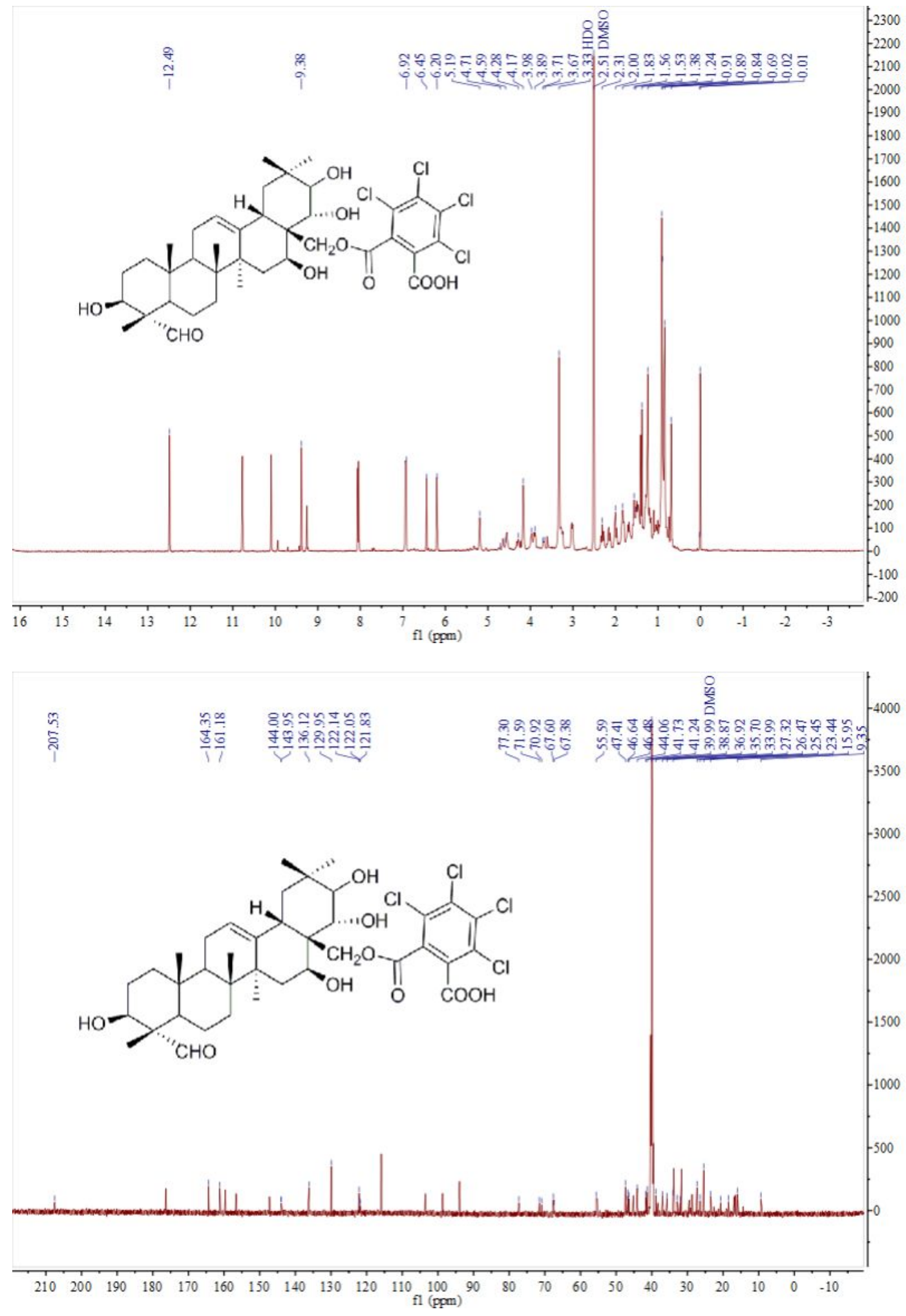




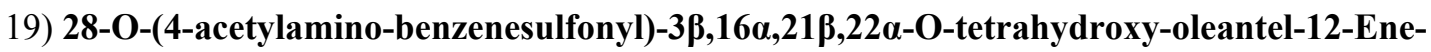
23-aldehyde (S-19)

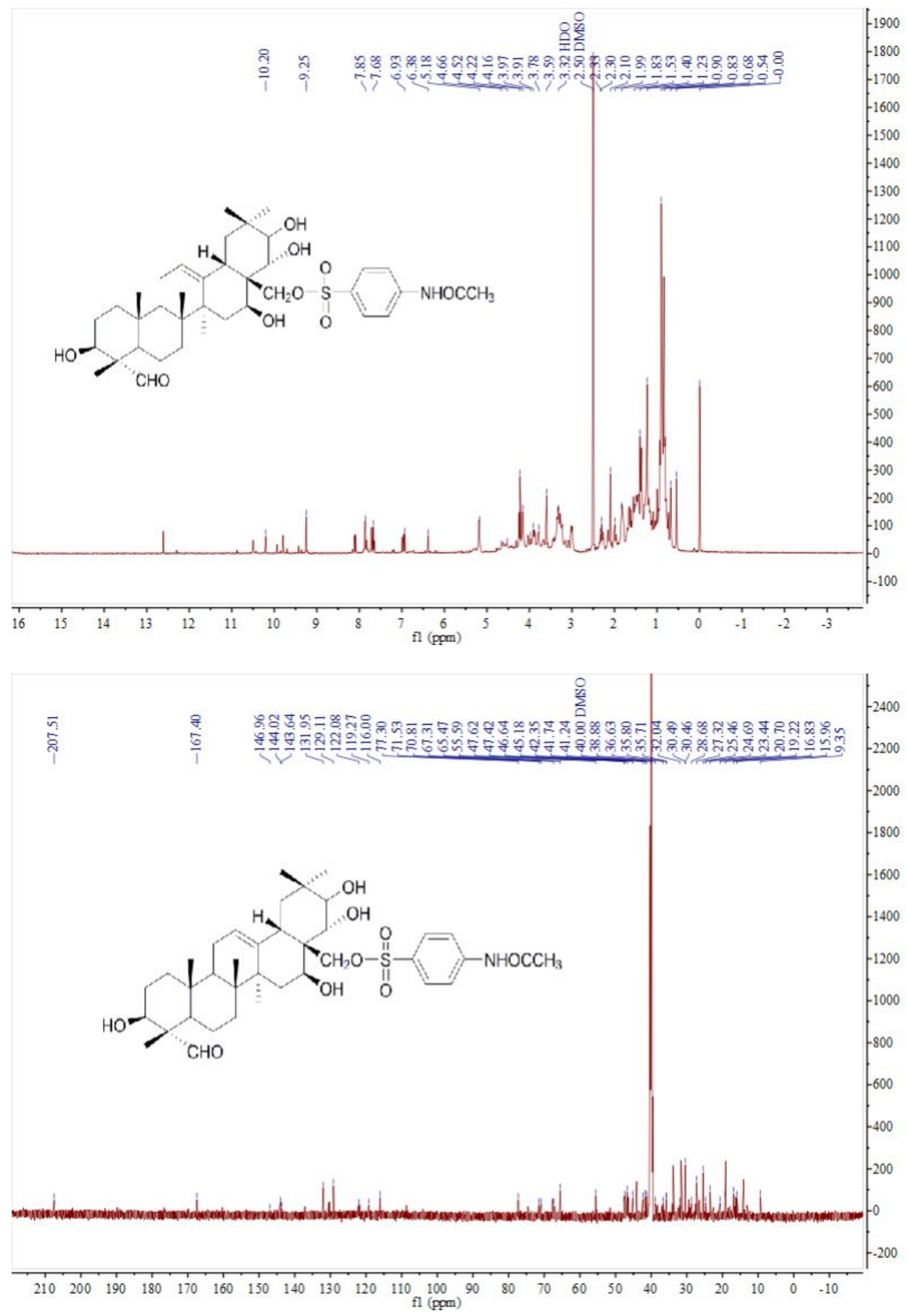

